\title{
MULTI-WAVELENGTH OBSERVATIONS OF 3FGL J2039.6-5618: A CANDIDATE REDBACK MILLISECOND
} PULSAR

\author{
D. Salvetti ${ }^{1}$, R. P. Mignani ${ }^{1,2}$, A. De Luca ${ }^{1,3}$, C. Delvaux ${ }^{4}$, C. Pallanca ${ }^{5}$, A. Belfiore ${ }^{1}$, M. Marelli ${ }^{1}$, A. A. Breeveld ${ }^{6}$, \\ J. Greiner ${ }^{4}$, W. Becker ${ }^{4}$, and D. Pizzocaro ${ }^{1,7}$ \\ ${ }^{1}$ INAF-Istituto di Astrofisica Spaziale e Fisica Cosmica Milano, via E. Bassini 15, I-20133, Milano, Italy \\ 2 Janusz Gill Institute of Astronomy, University of Zielona Góra, Lubuska 2, 65-265, Zielona Góra, Poland \\ ${ }^{3}$ Istituto Nazionale di Fisica Nucleare, Sezione di Pavia, Via Bassi 6, I-27100 Pavia, Italy \\ ${ }^{4}$ Max-Planck Institut für Extraterrestrische Physik, Giessenbachstrasse 1, D-85741 Garching bei München, Germany \\ ${ }^{5}$ Dipartimento di Fisica e Astronomia, Università degli Studi di Bologna, Viale Berti Pichat 6-2, I-40127, Bologna, Italy \\ ${ }^{6}$ Mullard Space Science Laboratory, University College London, Holmbury St. Mary, Dorking, Surrey, RH5 6NT, UK \\ ${ }^{7}$ Universitá degli Studi dell'Insubria, Via Ravasi 2, I-21100 Varese, Italy \\ Received 2015 September 25; accepted 2015 October 15; published 2015 November 19
}

\begin{abstract}
We present multi-wavelength observations of the unassociated $\gamma$-ray source 3FGL J2039.6-5618 detected by the Fermi Large Area Telescope. The source $\gamma$-ray properties suggest that it is a pulsar, most likely a millisecond pulsar, for which neither radio nor $\gamma$-ray pulsations have been detected. We observed 3FGL J2039.6-5618 with $X M M$-Newton and discovered several candidate X-ray counterparts within/close to the $\gamma$-ray error box. The brightest of these X-ray sources is variable with a period of $0.2245 \pm 0.0081$ days. Its X-ray spectrum can be described by a power law with photon index $\Gamma_{\mathrm{X}}=1.36 \pm 0.09$, and hydrogen column density $N_{\mathrm{H}}<4 \times 10^{20} \mathrm{~cm}^{-2}$, which gives an unabsorbed $0.3-10 \mathrm{keV}$ X-ray flux of $1.02 \times 10^{-13} \mathrm{erg} \mathrm{cm}^{-2} \mathrm{~s}^{-1}$. Observations with the Gamma-Ray Burst Optical/Near-Infrared Detector discovered an optical counterpart to this X-ray source, with a time-averaged magnitude $\mathrm{g}^{\prime} \sim 19.5$. The counterpart features a flux modulation with a period of $0.22748 \pm 0.00043$ days that coincides, within the errors, with that of the X-ray source, confirming the association based on the positional coincidence. We interpret the observed X-ray/optical periodicity as the orbital period of a close binary system where one of the two members is a neutron star. The light curve profile of the companion star, which has two asymmetric peaks, suggests that the optical emission comes from two regions with different temperatures on its tidally distorted surface. Based upon its X-ray and optical properties, we consider this source as the most likely X-ray counterpart to 3FGL J2039.6-5618, which we propose to be a new redback system.
\end{abstract}

Key words: binaries: general - gamma rays: stars - pulsars: general - stars: variables: general

\section{INTRODUCTION}

The launch of the Fermi Gamma-ray Space Telescope in 2008 June marked a new era in $\gamma$-ray astronomy, thanks to the unprecedented performance of its Large Area Telescope (LAT; Atwood et al. 2009). The recently released Third Fermi-LAT $\gamma$ ray source catalog (3FGL; Acero et al. 2015) derived from the first four years of observations contains 3033 sources. About $70 \%$ of these sources have been either directly identified (e.g., from the detection of $\gamma$-ray pulsations, pulsars, or correlated $\gamma$ ray and optical/radio variability, active galactic nuclei, novae, $\mathrm{X}$-ray binaries), or associated with objects that are either known or potential $\gamma$-ray emitters. The remaining $30 \%$ of the 3FGL sources have yet to be associated with any object, hence they are referred to as unassociated, and their nature is unknown.

Because pulsars are the largest family of $\gamma$-ray sources identified in the Galaxy $\left(\sim 160\right.$ and counting $\left.{ }^{8}\right)$, a significant fraction of the unassociated Fermi-LAT sources might be $\gamma$-ray pulsars. Some may have no or extremely faint radio emission, and so far escaped detection in all radio pulsar surveys. Indeed, many of these radio-quiet, or radio faint, $\gamma$-ray pulsars have been discovered through blind periodicity searches in the $\gamma$-ray data (e.g., Abdo et al. 2009) using novel search techniques (e.g., Atwood et al. 2006; Pletsch et al. 2013). About 45\% of the $\gamma$-ray pulsars discovered by the Fermi-LAT are millisecond

\footnotetext{
8 https://confluence.slac.stanford.edu/display/GLAMCOG/Public+List + of +LAT-Detected+Gamma-Ray+Pulsars
}

pulsars (MSPs). Interestingly, the vast majority of these MSPs $(\sim 80 \%)$ are in binary systems. Some have an He white dwarf (WD) companion star of mass $0.1 M_{\odot} \lesssim M_{\mathrm{C}} \lesssim 0.5 M_{\odot}$, whereas others have an usually non-degenerate companion (a late main sequence star or a brown dwarf), which is ablated by irradiation from the pulsar wind. Two distinct families of binary MSPs are recognized depending on the degree of the ablation processes: the Black Widow (BW) MSPs, where the companion is a very low-mass star of $M_{\mathrm{C}} \lesssim 0.1 M_{\odot}$ almost fully ablated by the pulsar wind, and the redback (RB) MSPs, where the companion is only partially ablated and has an higher mass of $M_{\mathrm{C}} \sim 0.1-0.4 M_{\odot}$ (Roberts 2013).

The use of automatic classification codes (e.g., Ackermann et al. 2012; Lee et al. 2012; Mirabal et al. 2012) based on the $\gamma$ ray characteristics is crucial to single out pulsar candidates among the many unassociated Fermi-LAT sources and optimize a systematic search for new $\gamma$-ray pulsars. Because $\gamma$-ray pulsars are also identified in the optical and X-rays (e.g., Abdo et al. 2013), multi-wavelength follow-ups of unidentified Fermi-LAT sources are still key to confirm the proposed pulsar classifications. In particular, optical observations are an important aid in the search for binary MSPs, for which blind periodicity searches in $\gamma$-rays must account for the unknown orbital parameters, requiring a massive use of super-computing power facilities (Pletsch \& Clark 2014). Optical observations identified the two Fermi-LAT sources 2FGL J2339.7-0531 and 2FGL J1311.7-3429 as binary MSPs prior to the 
detection of radio or $\gamma$-ray pulsations (Pletsch et al. 2012; Ray et al. 2013; P. Ray et al. 2015, in preparation) through the discovery of orbital modulations in the flux of their companion stars (Romani \& Shaw 2011; Kong et al. 2012; Kataoka et al. 2012; Romani et al. 2012). Similarly, new binary MSP candidates have been identified for the two unassociated Fermi-LAT sources: 2FGL J1653.6-0159 (Kong et al. 2014; Romani et al. 2014) and 2FGL J0523.3-2530 (Strader et al. 2014).

We studied 3FGL J2039.6-5618 as part of a pilot project aimed at identifying different classes of unassociated FermiLAT sources. This is a moderately bright $\gamma$-ray source (detection significance $\sim 25 \sigma$ ) that was listed in both the First (Abdo et al. 2010) and Second (Nolan et al. 2012) Fermi-LAT $\gamma$-ray source catalogs (a.k.a., 1FGL J2039.4-5621 and 2FGL J2039.8-5620, respectively), but has remained unassociated ever since. The 3FGL J2039.6-5618 field was observed in Xrays for the first time with the Swift/X-Ray Telescope (XRT) during snapshot observations ( 1 and $3.6 \mathrm{ks}$ exposure times) but no candidate X-ray counterpart was detected within the 2FGL $\gamma$-ray source error circle (Takeuchi et al. 2013). In radio, no potential counterpart was found in the Sydney University Molonglo Sky Survey source catalog (Mauch et al. 2003) or in dedicated observations of unassociated 2FGL sources with the Australia Telescope Compact Array (Petrov et al. 2013) and the Parkes radio telescope (Camilo et al. 2015). At very high energies 3FGL J2039.6-5618 is not associated with any known TeV source. ${ }^{9}$ Based on its $\gamma$-ray characteristics, 3FGL J2039.6-5618 was classified as a very likely pulsar candidate by Mirabal et al. (2013). Our newly developed classification method (Salvetti et al. 2013; D. Salvetti et al. 2015, in preparation) confirms that 3FGL J2039.6-5618 is a very likely pulsar candidate and suggests it is probably an MSP that is either isolated or in a binary system (see Section 2.1). However, since $\sim 80 \%$ of the MSPs detected by the Fermi-LAT are in binary systems, one can expect that would be a binary MSP.

We investigated this scenario through a multi-wavelength observation campaign (X-rays, ultraviolet, optical, infrared) of 3FGL J2039.6-5618 carried out using both dedicated observations and exploiting data available in public archives. The observations and data reduction are described in Section 2 and the results are presented in Section 3. Discussion and conclusions follow in Sections 4 and 5, respectively.

\section{OBSERVATIONS AND DATA REDUCTION}

\subsection{Target Selection}

We recently developed an advanced classification code (Salvetti et al. 2013) that can recognize different classes of $\gamma$ ray pulsars (e.g., young/middle-aged pulsars and MSPs). This code uses a statistical predictive method based on Artificial Neural Network (ANN) techniques to quantify the probability of a given source to be MSP-like on the basis of its $\gamma$-ray temporal and spectral characteristics. The method is based on an advanced hierarchical ANN architecture consisting of simple neural networks applied in sequence to first discriminate pulsar-like from active galactic nucleus (AGN)-like objects and then disentangle MSPs from young/middle age pulsars. This method correctly classifies $84 \%$ of the identified MSPs, while

\footnotetext{
http://tevcat.uchicago.edu/
}

the false positive fraction is lower than $10 \%$ (D. Salvetti et al. 2015, in preparation). We then applied the optimized hierarchical neural network to all unassociated 3FGL sources, to rank them according to their MSP probability function. As a result, 3FGL J2039.6-5618 was classified as an MSP-like object with a probability greater than $99 \%$. Therefore, it stands out as an obvious candidate for multi-wavelength investigations.

\subsection{X-Ray Observations}

We carried out an XMM-Newton observation of the 3FGL J2039.6-5618 $\gamma$-ray error box (Programme ID: 0720750301), which started on 2013 October 10 at 09:43:18 UT (revolution 2534) and lasted $44.6 \mathrm{ks}$. The pn detector (Struder et al. 2001) of the European Photon Imaging Camera (EPIC) instrument was operated in Extended Full Frame mode, with a time resolution of $200 \mathrm{~ms}$ over a $26^{\prime} \times 27^{\prime}$ field of view (FOV), while the Metal Oxide Semi-conductor (MOS) detectors (Turner et al. 2001) were set in Full Frame mode (2.6 s time resolution on a $15^{\prime}$ radius FOV). The thin optical filter was used for the pn, while a medium one was used for the MOS cameras. We retrieved the Observation Data Files from the XMMNewton Science Archive ${ }^{10}$ and used the most recent release of the XMM-Newton Science Analysis Software (SAS) v14.0 to analyze them. We performed standard data processing with the epproc and emproc tools, and screened for high particle background time intervals (e.g., De Luca et al. 2005). Our analysis revealed no significant contamination from soft protons. After the standard data processing, the good, deadtime corrected exposure time was $41.6 \mathrm{ks}$ for the pn and $43.2 \mathrm{ks}$ for the two MOS detectors.

\subsection{Ultraviolet, Optical, Infrared Observations}

In the optical/near-infrared (near-IR), we observed the 3FGL J2039.6-5618 field with the Gamma-Ray Burst Optical/Near-Infrared Detector (GROND; Greiner et al. 2008) at the MPI/ESO $2.2 \mathrm{~m}$ telescope on La Silla (Chile). The field was repeatedly observed on 2014 August 16, 17 , and 18 in the $\mathrm{g}^{\prime}, \mathrm{r}^{\prime}, \mathrm{i}^{\prime}$, and $\mathrm{z}^{\prime}$ bands in the optical, and in the $\mathrm{J}, \mathrm{H}$, and $\mathrm{K}_{\mathrm{s}}$ bands in the near-IR. The observations were split into sequences of 18,18 , and 17 exposures per day, each consisting of four $115 \mathrm{~s}$ dithered exposures in the optical and forty-eight $10 \mathrm{~s}$ dithered exposures in the near-IR. The observations were executed in gray time with airmass between 1.12 and 1.28 and mean seeing of $1{ }^{\prime \prime} 0$. Single dithered exposures were reduced (bias subtraction, flat-fielding, distortion correction) and stacked using standard IRAF $^{11}$ tasks implemented in the GROND pipeline (Krühler et al. 2008; Yoldas et al. 2008). The astrometry calibration was computed on single exposures against stars selected from the USNO-B1.0 catalog (Monet et al. 2003) in the optical bands and the 2MASS catalog (Skrutskie et al. 2006) in the near-IR bands, yielding an accuracy of $0 . ! 3$ with respect to the chosen reference frame. The photometric calibration in the optical was computed against a close-by field from the Sloan Digital Sky Survey (York et al. 2000) at $\delta=-10^{\circ}$, observed in the first night

\footnotetext{
${ }^{10} \mathrm{http}: / / \mathrm{xmm}$. esac.esa.int/xsa/

11 IRAF is distributed by the National Optical Astronomy Observatories, which are operated by the Association of Universities for Research in Astronomy, Inc., under cooperative agreement with the National Science Foundation.
} 
under photometric conditions. From the calibrated images, we extracted a grid of secondary photometric calibrators for direct on-the-frame calibration on the subsequent nights. In the nearIR, the photometric calibration was computed against 2MASS stars identified in the GROND field of view. The accuracy of the absolute photometry calibration was $0.02 \mathrm{mag}$ in the $\mathrm{g}^{\prime}, \mathrm{r}^{\prime}$, $\mathrm{i}^{\prime}, \mathrm{z}^{\prime}$ and bands; 0.03 mag in $\mathrm{J}$ and $\mathrm{H}$ bands; and 0.05 in the $\mathrm{K}_{\mathrm{s}}$ band.

In addition to GROND, we used serendipitous $\mathrm{JHK}_{\mathrm{s}}$ images of the 3FGL J2039.6-5618 field taken on 2010 July 16 and 25 with the near-IR camera (VIRCAM; Dalton et al. 2006) of the Visible and Infrared Survey Telescope for Astronomy (VISTA; Emerson et al. 2006). The data set, which was processed and calibrated at the Cambridge Astronomical Survey Unit $\left(\mathrm{CASU}^{12}\right)$, consists of three sequences of 16 consecutive exposures of $15 \mathrm{~s}$ each in the $\mathrm{J}$ band and of $7.5 \mathrm{~s}$ each in both the $\mathrm{H}$ and $\mathrm{K}_{\mathrm{s}}$ bands. In the optical and near-ultraviolet (nearUV) we used U, UVW1 $(\lambda=2910 \AA ; \Delta \lambda=1180 \AA)$, and UVM2 ( $\lambda=2310 \AA ; \Delta \lambda=710 \AA)$ images from the $X M M$ Newton Optical Monitor (OM; Mason et al. 2001) that were obtained in parallel to our observations with exposure times of 1900,2700 , and $3080 \mathrm{~s}$, respectively. We also used archival UVW2 $(\lambda=2055 \AA ; \Delta \lambda=557 \AA)$ images from the Swift UltraViolet and Optical Telescope (UVOT; Roming et al. 2005) performed on 2011 February 21 $($ OBSID $=00041479002)$, consisting of five exposures for a total integration time of $3587 \mathrm{~s}$. The OM and UVOT data were processed and calibrated using the SAS tool omichain and the HEASOFT software package, respectively.

\section{DATA ANALYSIS AND RESULTS}

To search for possible counterparts of 3FGL J2039.6-5618 in our multi-wavelength observations, we used its recent 3FGL position as a reference (Acero et al. 2015): $\alpha=20^{\mathrm{h}} 39^{\mathrm{m}} 40^{\mathrm{s}} 32$ and $\delta=-56^{\circ} 18^{\prime} 43 !^{\prime \prime} 6$ (J2000). Its associated 95\% confidence position error ellipse has a semimajor and semiminor axis of $2 ! 6$ and $2 ! 4$, respectively, and a position angle of $74^{\circ} .95$, which is measured east of north. We started from our XMM-Newton observations to find potential X-ray counterparts to 3FGL J2039.6-5618. Then we used our multi-wavelength database to single out those that are most likely associated with this $\gamma$ ray source. In particular, because we expect that 3FGL J2039.6 -5618 is a binary MSP, we focused our analysis on X-ray sources that show variability and/or have variable optical counterparts, possibly featuring periodic flux modulations.

\subsection{X-Ray Data Analysis}

\subsubsection{Source Detection}

For our X-ray analysis, we selected only 0-4 pattern events from the pn and 0-12 from the two MOS detectors with the default flag mask. The source detection in the $0.3-10 \mathrm{keV}$ energy range was run simultaneously on the event lists of each EPIC-pn and MOS detector using a maximum likelihood fitting with the SAS task edetect_chain invoking other SAS tools to produce background, sensitivity, and vignetting-corrected exposure maps. The final source list includes $90 \mathrm{X}$-ray sources from both the pn and MOS detectors, with a combined pn + MOS detection likelihood greater than 10, corresponding to a significance above $3.5 \sigma$. Figure 1 shows the $0.3-10 \mathrm{keV}$

\footnotetext{
12 http://casu.ast.cam.ac.uk/
}

exposure-corrected XMM-Newton FOV that was obtained combining the images of the EPIC-pn and MOS detectors. We focused our analysis on the $16 \mathrm{X}$-ray sources detected within, or close to, the $95 \%$ confidence position error ellipse of 3FGL J2039.6-5618. We summarized the positions, spectral parameters, fluxes, and variability indices of these sources in Table 1.

\subsubsection{Spectral Analysis}

For each EPIC detector, we extracted the source photons using an extraction radius of $20^{\prime \prime}$, while we extracted background photons from source-free regions in the same CCD chip as the source, with radii of $50^{\prime \prime}-120^{\prime \prime}$. For each detector, we used the SAS task specgroup to rebin all the extracted spectra and have at least 25 counts for each background-subtracted spectral channel, as well as generated ad hoc response matrices and ancillary files using the SAS tasks rmfgen and arfgen. For each source, we fitted simultaneously the pn and MOS spectra using XSPEC v12.8, forcing the same set of parameters and considering three different spectral models: a power law (PL)—which is well suited for both AGN and pulsars-an apec (AP) for stellar coronae, and a blackbody (BB) for the pulsar thermal component. In all cases, the hydrogen column density $N_{\mathrm{H}}$ was left as a free parameter. For each emission model we computed the $90 \%$ confidence level error on the spectral parameters. When the best-fit $N_{\mathrm{H}}$ values were comparable to zero, we assumed the measured uncertainties to determine the $3 \sigma$ confidence level upper limit. Spectra with very low counts were fitted after fixing the $N_{\mathrm{H}}$ to the estimated value along the line of sight $\left(5 \times 10^{20} \mathrm{~cm}^{-2}\right.$; Dickey \& Lockman 1990), or fixing either the photon index or the temperature to typical values (i.e., $\Gamma_{\mathrm{X}}=2$ and $k T=3.5 \mathrm{keV}$ or $k T=0.2 \mathrm{keV}$ in case of an AP and a BB model, respectively).

As shown in Table 1, only Source 3 and 11, the two brightest $\mathrm{X}$-ray sources in our sample, were best fitted by a simple PL model, with a $\chi^{2}=52.32$ (42 degrees of freedom, dof) for the former and $\chi^{2}=16.06$ (22 dof) for the latter. For the remaining 13 sources, at least two different models were required to obtain an acceptable fit with null hypothesis probability $>0.01$. Only for Source 88 was it not possible to obtain acceptable results with any of the selected spectral models. Indeed, this source is the faintest in our sample and is likely spurious. Figure 2 shows the binned spectrum of Source 3 extracted simultaneously from each of the EPIC detectors, together with the best-fit PL model. Since most MSPs are characterized by a significant thermal component to their X-ray spectra, we tried to fit the spectra of Source 3 and Source 11 with an absorbed BB plus PL model. This improved the accuracy of the fit only for Source 3 , with a $\chi^{2}=34.9$ (40 dof). From the F-test (Bevington 1969), we computed a 0.0003 probability that adding a thermal component to the model would produce a chance improvement to the fit. Since this probability only corresponds to a $\sim 3.6 \sigma$ significance, hereafter we ignored the absorbed BB plus PL spectral model. Because the measured counts are too few to clearly discriminate among different spectral models in most X-ray sources, we checked whether we could extract qualitative spectral information from an hardness ratio (HR) analysis (Marelli et al. 2014). However, we found that the observed HRs are compatible with different spectral models and, therefore, are not constraining. 


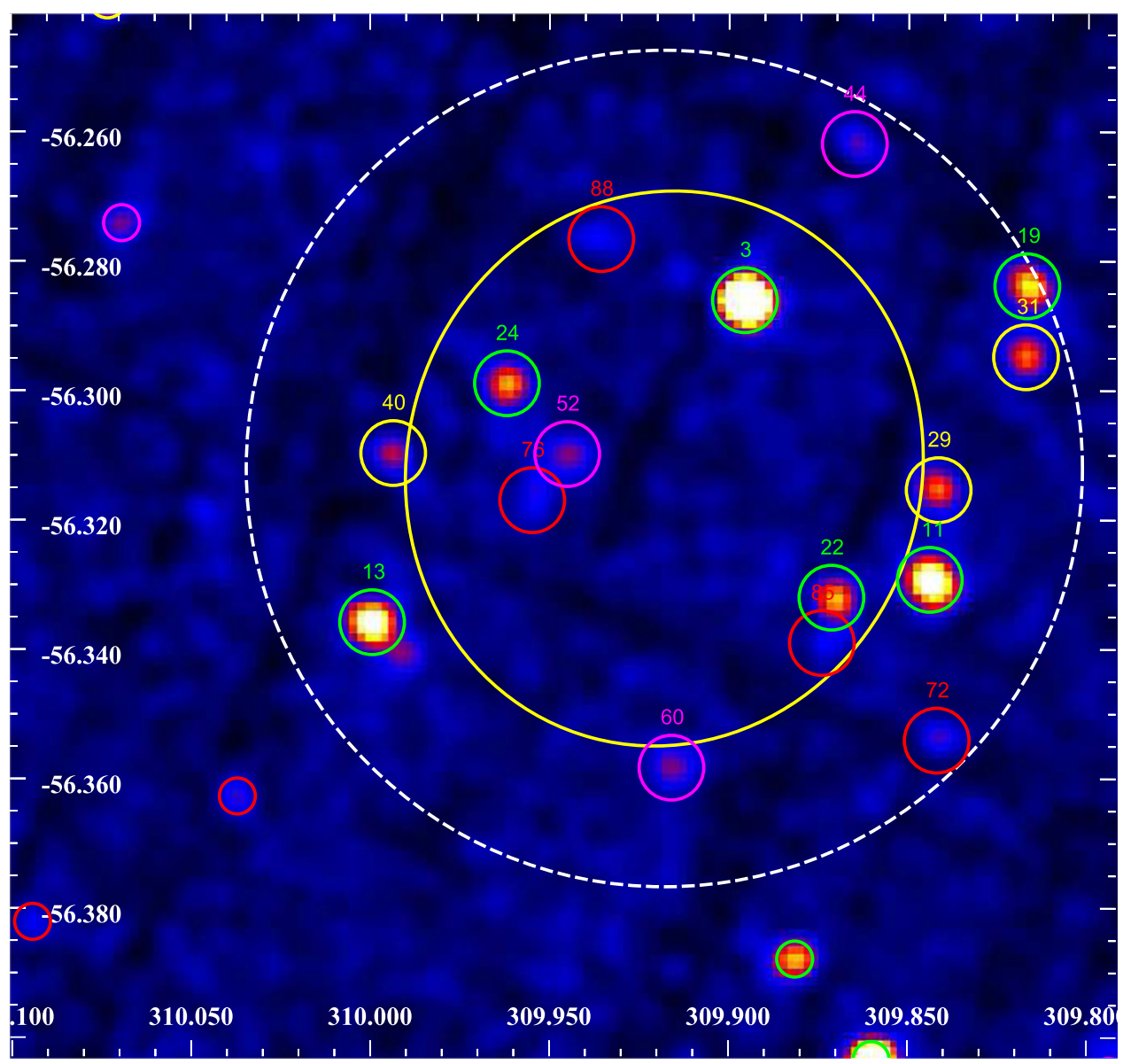

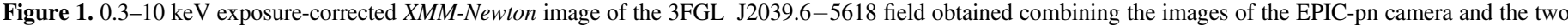

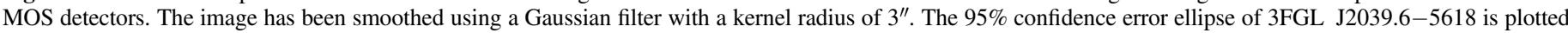

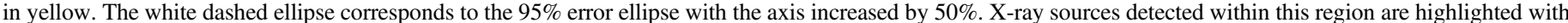

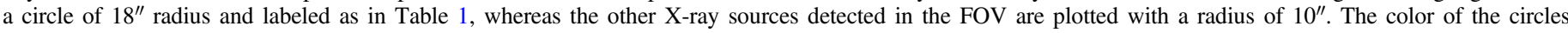

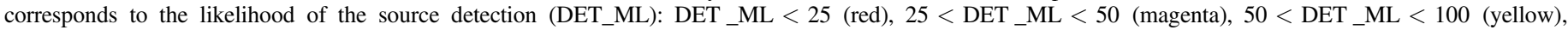
DET_ML > 100 (green).

\subsubsection{Variability Analysis}

In order to detect possible time variability during the $X M M$ Newton observation, we generated standard light curves from the pn-data for all $16 \mathrm{X}$-ray sources in Table 2. Starting from the source and background regions described in Section 3.1.2, we extracted source+background and background light curves, respectively, with the SAS task evselect and combined them into a background-subtracted light curve with the task epiclccorr. This task also corrects the time series for vignetting, bad pixels, chip gaps, quantum efficiency, dead time, exposure, and good time intervals. For each source, we generated a light curve with time binning of $2500 \mathrm{~s}$, or multiple of this value, to have at least 25 counts per bin and we ran an $\chi^{2}$ test to evaluate the variability significance. Only Source 3 is characterized by a significant variability during the observation $\left(\chi^{2}=66.18\right.$, with $\left.16 \mathrm{dof}\right)$, with a chance probability of $4.61 \times 10^{-8}(>5 \sigma)$. After combining the data from all three EPIC cameras, the Source 3 light curve shows a more apparent variability (Figure 3, left), with a chance probability of $7.43 \times 10^{-14}$, whereas the other X-ray still shows no evidence of significant variability.

The Source 3 light curve also hints at a possibly periodic modulation. We converted photon arrival times to the Solar system Barycentric Dynamical Time with the SAS task barycen and used the FTOOL task efsearch to find the best period in the light curve through a maximum $\chi^{2}$ test. We folded the light curve with periods ranging from 100 to $43,000 \mathrm{~s}$, with the latter comparable to the length of the $X M M$ Newton observation. We found the best-fit period at $0.2245 \pm 0.0081$ days, where the period uncertainty was obtained following Leahy (1987). The corresponding $\chi^{2}$ of 104.5 (9 dof) gives a chance probability of $6.38 \times 10^{-14}$, accounting for the number of trials, and makes the periodicity statistically significant $(\sim 7.5 \sigma)$. The presence of a periodic signal at the corresponding frequency of $\sim 5 \times 10^{-5} \mathrm{~Hz}$ was independently confirmed by the power spectrum produced with the FTOOL powspec. We note that the best-fit X-ray period of Source 3 is comparable to about half the length of the XMMNewton observation, so that the observed periodicity might be spurious. We examined the light curves of other comparably bright X-ray sources detected in the whole XMM-Newton FOV and found that none showed evidence of periodicity at any timescale. Nonetheless, the fact that the length of the $X M M$ Newton observation only covers $\sim 2.3$ cycles prevents us from firmly claiming that Source 3 is periodic. The X-ray light curve folded at the best-fit period (Figure 3, right) is characterized by 
Table 1

Spectral Properties of the XMM-Newton Sources Detected within, or Close to, the Error Ellipse of 3FGL J2039.6-5618. Spectral Models are: Power-law (PL), apec (AP), and Blackbody (BB)

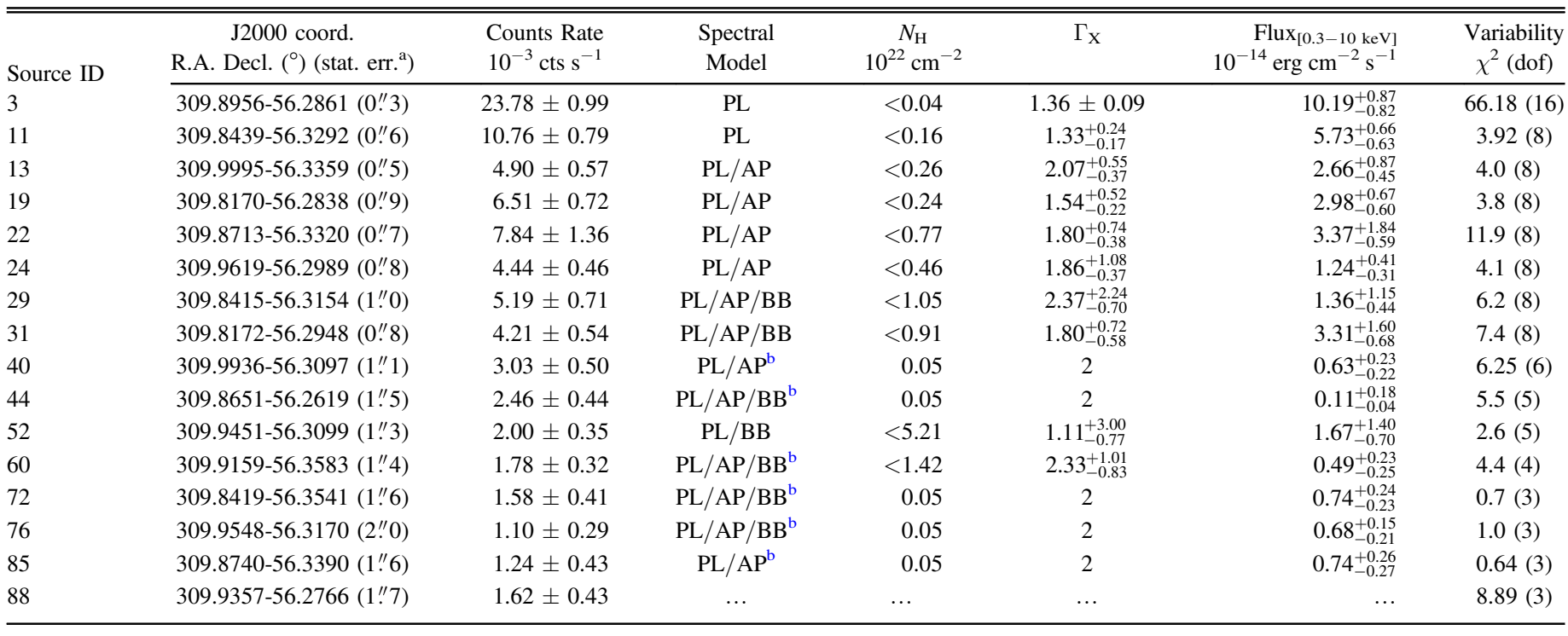

Notes. Results of the spectral analysis of the XMM-Newton sources detected within an error circle of 1.5 times the 95\% confidence error ellipse of 3FGL J2039.6 -5618. If the spectrum is well fitted by more than one model, we report only the PL parameters. Here, we report the best-fit X-ray position, the count rate of the bestfit spectral model, the best-fit column density, the best-fit photon index, the unabsorbed X-ray flux in the $0.3-10 \mathrm{keV}$ energy band, and the variability test described in the text. The errors are at a $90 \%$ confidence and the upper limits at $3 \sigma$.

${ }^{a}$ Here we report only the $1 \sigma$ statistical error, the $1 \sigma$ systematic error is about 1 ." 5 for each X-ray source.

${ }^{\mathrm{b}}$ Owing to the low number of counts, we fixed the photon index $\left(\Gamma_{\mathrm{X}}\right)$ to 2 for a PL model and the temperature $(k T)$ to 3.5 and 0.2 for an AP and a BB model, respectively.

two peaks separated in phase by $0.31 \pm 0.04$. Therefore, it cannot be described by a simple sinusoidal or Gaussian model, with a null hypothesis probability lower than $10^{-3}$. We checked whether the folded X-ray light curve of Source 3 varied as a function of the energy, and whether the X-ray spectrum changed as a function of the phase. In both cases, however, the available statistics is not sufficient to highlight significant differences in the energy-resolved light curves and the phase-resolved spectra.

We looked for archival X-ray images of the 3FGL J2039.6 -5618 field to check for long-term variability of Source 3 . The field was serendipitously observed with the X-ray Imaging Spectrometer (XIS) of Suzaku (Mitsuda et al. 2007) on 2010 October 28 for a total exposure time of $21.5 \mathrm{ks}$ (OBSID 705028010). We extracted source counts from a circle of radius 1.3 around the best XMM-Newton coordinates of Source 3 and background counts from a nearby, source-free $2^{\prime}$-radius circle using the HEASOFT(v.6.16) tool xselect, and summed the spectra from the XIS cameras with the mathpha, addarf, and addrmf tools. We obtained 350 counts, of which $\sim 50 \%$ are from the source. A fit with a PL gives a null hypothesis probability of 0.06 (4 dof), with $N_{\mathrm{H}}<5 \times 10^{21} \mathrm{~cm}^{-2}$ (90\% upper limit), photon index $\Gamma=1.3 \pm 0.4$, and an unabsorbed flux in the $0.3-10 \mathrm{keV}$ energy range of $1.7_{-1.0}^{+0.3} \times 10^{-13} \mathrm{erg} \mathrm{cm}^{-2} \mathrm{~s}^{-1}$, which is fully compatible with the XMM-Newton results. The XMM-Newton count-rate and spectral parameters (Table 1) are also compatible with nondetection above the $3 \sigma$ threshold $\left(\sim 1.05 \times 10^{-13} \mathrm{erg} \mathrm{cm}^{-2} \mathrm{~s}^{-1}\right)$ of Source 3 in the short Swift/XRT images of Takeuchi et al. (2013), taken in 2010 and 2011. Therefore, we find no evidence of variability of Source 3 on timescales of three years.

\subsection{IR/Optical/UV Analysis}

\subsubsection{Source Cross-identification}

We cross-matched the positions of all the 16 XMM-Newton sources in Table 1 with the source catalogs obtained from the GROND observations. We performed the source detection on the single-band GROND exposures using the starfind tool in IRAF, matched the source catalogs over the different observations, and checked for variable sources against the median of all observations. Object photometry was computed using the task daophot in IRAF and the airmass correction was applied using the standard atmospheric extinction coefficients for the La Silla Observatory. For the cross-match we used a radius obtained by combining the statistical $1 \sigma$ uncertainty on the X-ray source centroid, plus the $90 \%$ confidence level systematic error associated with the absolute accuracy of the $X M M-N e w t o n$ aspect solution, which is $1 .{ }^{\prime \prime} 5$ per coordinate. ${ }^{13}$ The uncertainty on the absolute astrometry of the GROND images ( $(0$ ". 3$)$ is much lower than the XMM-Newton, and is accounted for by our choice of the matching radius. There are only six XMM-Newton sources (Source 3, 13, 22, 24, 40, 76) with at least a candidate optical or near-IR counterpart in the GROND data (Figure 4).

We also cross-correlated the XMM-Newton source list with the VISTA, OM, and UVOT source catalogs. The source detection and photometry in the VISTA images were carried out as part of the CASU pipeline (Section 2.3). For the OM images, the source detection and photometry were carried out with the SAS tasks omdetect and ommag, respectively, and source detection and photometry for the UVOT images were

$\overline{13}$ Calibration technical note XMM-SOC-CAL-TN-0018. 
Table 2

Magnitudes of the Optical, Near-IR, and Near-UV Counterparts to the XMM-Newton Sources Detected within, Close to, the Error Ellipse of 3FGL J2039.6-5618 (Table 1), Derived from the GROND, VISTA, OM, and UVOT Observations

\begin{tabular}{|c|c|c|c|c|c|c|c|c|c|c|c|c|c|c|}
\hline \multirow{2}{*}{ ID } & \multicolumn{7}{|c|}{ GROND } & \multicolumn{3}{|c|}{ VISTA } & \multicolumn{3}{|c|}{$\mathrm{OM}$} & \multirow{2}{*}{$\begin{array}{l}\text { UVOT } \\
\text { UVW2 }\end{array}$} \\
\hline & $\mathrm{g}^{\prime}$ & $\mathrm{r}^{\prime}$ & $\mathrm{i}^{\prime}$ & $\overline{\mathrm{z}^{\prime}}$ & $\mathrm{J}$ & $\mathrm{H}$ & $\mathrm{K}$ & $\mathrm{J}$ & $\mathrm{H}$ & $\mathrm{K}_{\mathrm{s}}$ & $\mathrm{U}$ & UVW1 & $\overline{\mathrm{UVM} 2}$ & \\
\hline 3 & 19.40 & 18.73 & 18.72 & 18.61 & 18.53 & 18.15 & 18.34 & 18.24 & 18.24 & 18.64 & $\ldots$ & 21.26 & $\ldots$ & 21.88 \\
\hline 11 & $\ldots$ & $\ldots$ & $\ldots$ & $\ldots$ & $\ldots$ & $\ldots$ & $\ldots$ & 20.33 & 20.02 & 20.73 & $\ldots$ & $\ldots$ & $\ldots$ & $\ldots$ \\
\hline 13 & $\ldots$ & $\ldots$ & 22.61 & $\ldots$ & $\ldots$ & $\ldots$ & $\ldots$ & $\ldots$ & $\ldots$ & 20.70 & $\ldots$ & 21.27 & $\ldots$ & $\ldots$ \\
\hline 19 & $\ldots$ & $\ldots$ & $\ldots$ & $\ldots$ & $\ldots$ & $\ldots$ & $\ldots$ & $\ldots$ & $\ldots$ & 20.64 & $\ldots$ & $\ldots$ & $\ldots$ & $\ldots$ \\
\hline \multirow[t]{2}{*}{22} & $\ldots$ & $\ldots$ & $\ldots$ & $\ldots$ & 19.37 & $\ldots$ & 17.88 & $\ldots$ & $\ldots$ & $\ldots$ & $\ldots$ & $\ldots$ & $\ldots$ & $\ldots$ \\
\hline & $\ldots$ & $\ldots$ & $\ldots$ & $\ldots$ & 18.02 & 17.96 & $\ldots$ & 17.84 & 17.93 & 17.97 & $\ldots$ & $\ldots$ & $\ldots$ & $\ldots$ \\
\hline 24 & 24.21 & $\ldots$ & $\ldots$ & $\ldots$ & $\ldots$ & $\ldots$ & $\ldots$ & $\ldots$ & $\ldots$ & $\ldots$ & $\ldots$ & $\ldots$ & $\ldots$ & $\ldots$ \\
\hline 29 & $\ldots$ & $\ldots$ & $\ldots$ & $\ldots$ & $\ldots$ & $\ldots$ & $\ldots$ & $\ldots$ & $\ldots$ & $\ldots$ & 20.83 & 20.39 & $\ldots$ & $\ldots$ \\
\hline 40 & 21.95 & 21.22 & 21.45 & 22.92 & $\ldots$ & $\cdots$ & $\cdots$ & $\cdots$ & 19.82 & 19.85 & 20.10 & 22.08 & $\ldots$ & $\ldots$ \\
\hline 72 & $\ldots$ & $\ldots$ & $\ldots$ & $\ldots$ & $\ldots$ & $\cdots$ & $\cdots$ & $\cdots$ & 19.66 & $\ldots$ & $\ldots$ & $\ldots$ & $\ldots$ & $\ldots$ \\
\hline 60 & $\ldots$ & $\ldots$ & $\ldots$ & $\ldots$ & $\ldots$ & $\ldots$ & $\ldots$ & $\ldots$ & $\ldots$ & $\ldots$ & $\ldots$ & 19.33 & $\ldots$ & $\ldots$ \\
\hline \multirow[t]{3}{*}{76} & 19.64 & 18.36 & 18.63 & 18.20 & 17.65 & 17.66 & 17.77 & 17.63 & 17.58 & 17.90 & $\ldots$ & $\ldots$ & $\ldots$ & $\ldots$ \\
\hline & $\ldots$ & $\ldots$ & $\ldots$ & 21.52 & $\ldots$ & $\ldots$ & $\ldots$ & 19.36 & 18.84 & 18.64 & $\ldots$ & $\ldots$ & $\ldots$ & $\ldots$ \\
\hline & $\ldots$ & $\ldots$ & $\ldots$ & 23.97 & $\ldots$ & $\ldots$ & $\ldots$ & $\ldots$ & $\ldots$ & $\ldots$ & $\ldots$ & $\ldots$ & $\ldots$ & $\ldots$ \\
\hline 88 & $\ldots$ & $\ldots$ & $\ldots$ & $\ldots$ & $\ldots$ & $\ldots$ & $\ldots$ & 20.93 & 21.31 & $\ldots$ & $\ldots$ & $\ldots$ & $\ldots$ & $\ldots$ \\
\hline
\end{tabular}

Note. For GROND we report the average magnitudes only. The matches have been computed using a radius of $3^{\prime \prime}$, which accounts for both the statistical and systematic errors on the XMM-Newton source coordinates (see Table 2) and the accuracy of the absolute astrometry of the optical, near-IR, and near-UV images. All magnitudes are in the $\mathrm{AB}$ system.

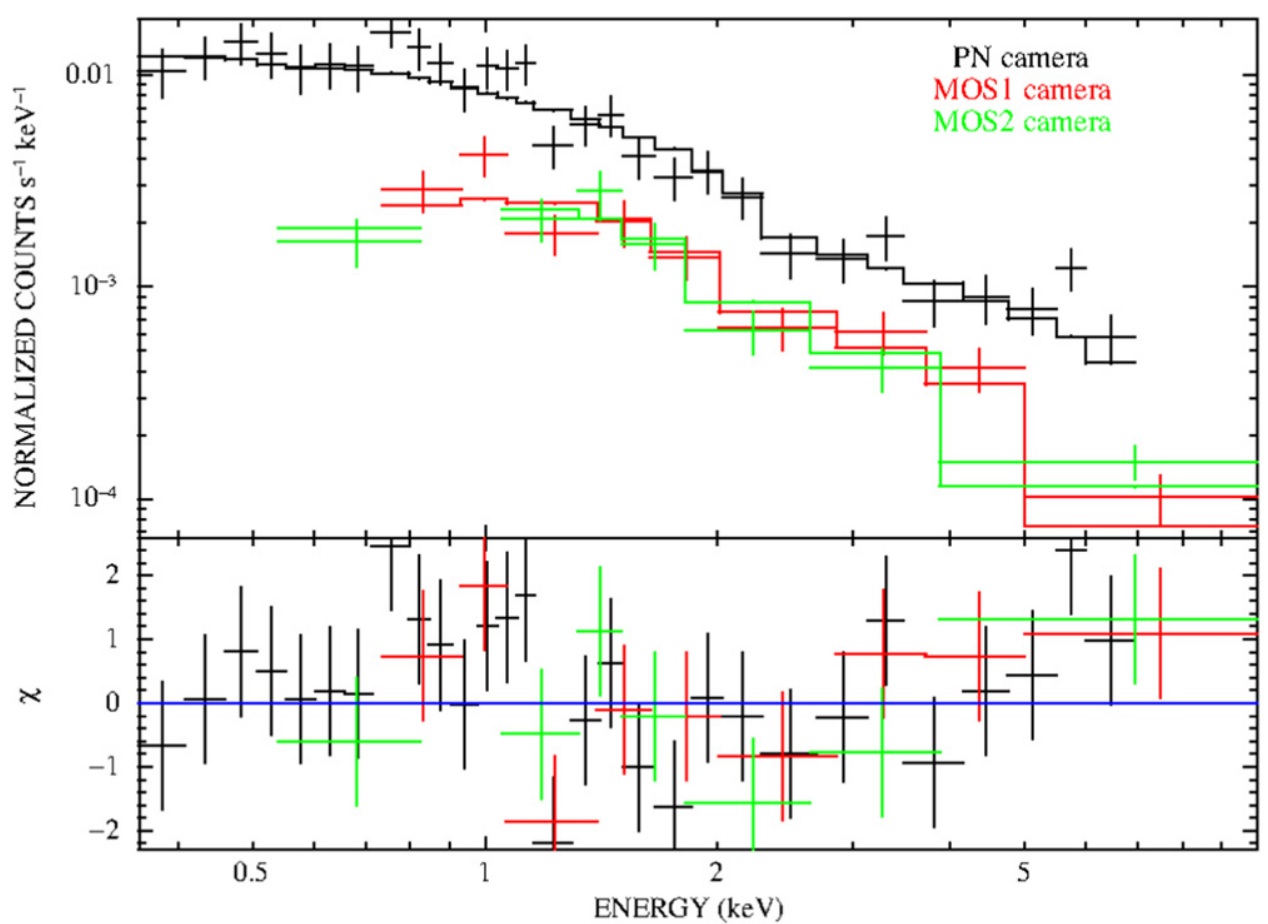

Figure 2. Binned spectrum of Source 3, the brightest source among the most probable candidate X-ray counterparts to 3FGL J2039.6-5618, obtained with each of the EPIC detectors and best-fit with PL models.

carried out with the HEASOFT taks uvotdetect and uvotsource. As before, the matching radius accounted for the uncertainty on the absolute astrometry of the used source catalogs: $\lesssim 0$ "! 3 (VISTA; Emerson et al. 2004), 0".5 (UVOT; Breeveld et al. 2010), and 0.7 (OM; Page et al. 2012). The cross-correlations with these catalogs provided additional nearIR and near-UV magnitudes for some of the GROND sources and unveiled candidate counterparts for Source 11, 19, 29, 60, and 88 . We found no counterparts in the OM/UVM2 filter, whereas only Source 3 was detected in the UVOT/UVW2 images. The optical, near-UV, and near-IR magnitudes of the candidate counterparts to the XMM-Newton sources are summarized in Table 2. Only for the candidate counterparts to Source 3, 40, and 76 do we have an adequate spectral coverage in at least the optical and near-IR.

\subsubsection{Variability Analysis}

Among the six XMM-Newton sources with a possible GROND counterpart, only Source 3 is associated with a 

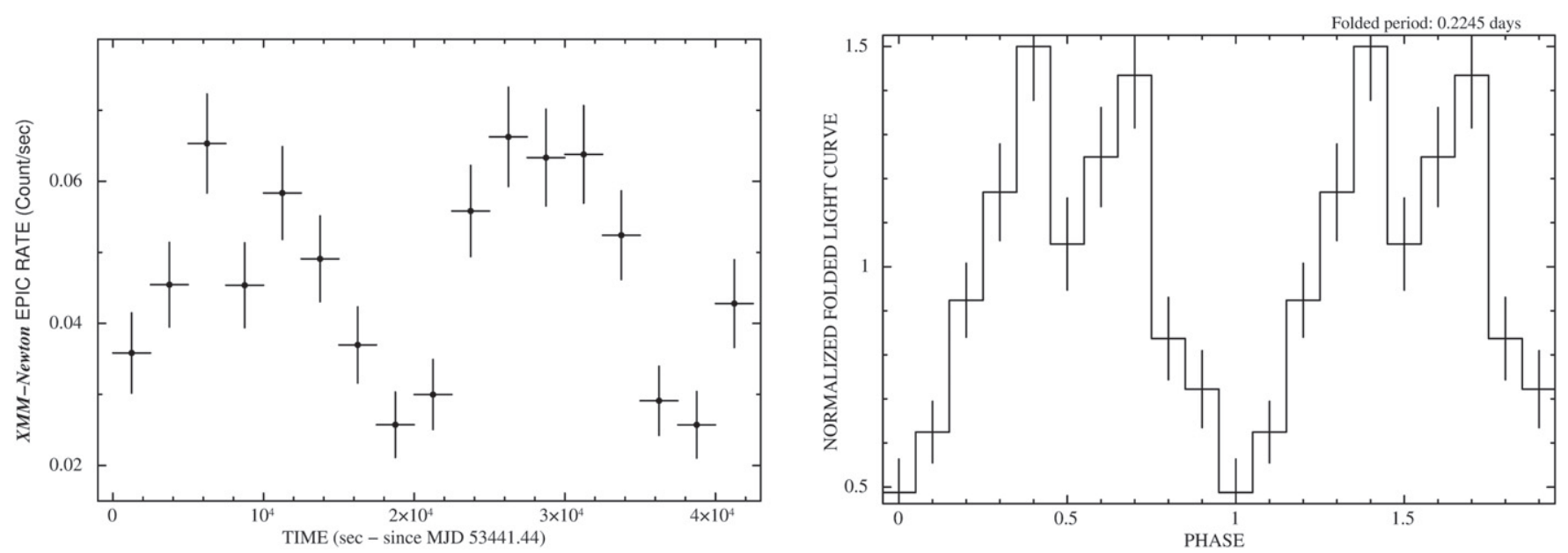

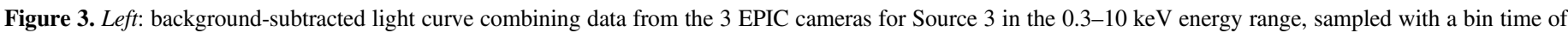
$2500 \mathrm{~s}$. Right: same, but folded around the best period of 0.2245 days normalized to the average source intensity. In both panels, error bars are reported at $1 \sigma$.

clearly variable object $\left(\chi^{2}=1232\right.$, with 51 dof). Figure 5 (left) shows the multi-band light curves of this object for the three nights spanned by the GROND observations. As seen, the light curves seem to be modulated, with an amplitude of $\sim 0.4 \mathrm{mag}$ in the $\mathrm{g}^{\prime}$ band. In particular, the modulation seems to be periodic and feature a double-peaked profile (night 1), with the two peaks only partially seen in night 2 and 3 , likely owing to the different sampling of the light curve. This modulation is also seen in the $\mathrm{r}^{\prime}, \mathrm{i}^{\prime}$, and $\mathrm{z}^{\prime}$ light curves, with both shape and amplitudes similar to that of $\mathrm{g}^{\prime}$, and is also recognized in the $\mathrm{J}$ and H-band light curves, and very marginally in the that of $\mathrm{K}$-band. This suggests that the observed modulation is real and not due, for instance, to possible problems with the photometry in a given filter. As a check, for all filters we extracted the light curves of several stars of comparable brightness detected in the GROND FOV, but found no evidence of such a modulation. This confirms that it is not due to random effects, such as variations in the sky conditions (transparency, background, moon illumination), or systematic effects, such as variations in the encircled flux due to the fixed size of the photometry aperture with respect to the seeing disk. Furthermore, the fact that the modulation seems to be periodic argues against the possibility that it is produced by any such effects, and implies that is associated with an intrinsic star variability.

We computed the probability that the association between Source 3 and its candidate GROND counterpart is due to a chance coincidence. We computed the probability as $P=1-\exp \left(-\pi \rho r^{2}\right)$, where $r$ is the matching radius used for Source $3\left(1{ }^{\prime \prime} 8\right)$ and $\rho$ is the density of stellar objects in the GROND field, regardless of their brightness, measured in the co-addition of all $\mathrm{g}^{\prime}$-band exposures. For a stellar density $\rho \sim 0.0019$ square degree ${ }^{-1}$ we estimated a chance coincidence probability $P \sim 0.02$, which makes a chance coincidence unlikely.

To confirm the existence of a periodic modulation of the Source 3 candidate counterpart, we carried out a periodicity analysis based on the Generalized Lombe-Scargle periodogram method (Lomb 1976; Scargle 1982; Zechmeister \& Kuerted 2009). To cross-check, we also used the phase dispersion minimization technique (Stellingwerf 1978) using the pdm code in IRAF and the Period0 4 software package (Lenz \& Breger 2005), which is especially dedicated to the statistical analysis of large astronomical time series-containing gaps (see Figure 5). All methods indicate the presence of a periodicity with a period of $\sim 0.227$ days. The analysis of the power spectrum of the optical time series shows a clear peak at the corresponding frequency, with a probability that is due to a chance noise fluctuation (false-alarm probability) of $\sim 1.8 \times 10^{-15}(\sim 8 \sigma)$. The best period was found at $0.22748 \pm 0.00043$ days in the optical bands and at $0.22799 \pm 0.00062$ days in the near-IR bands, where we estimated the period uncertainty following Gilliland \& Baliunas (1987). The optical and near-IR best-fit periods of the Source 3 candidate counterpart are consistent within the uncertainties, which provides further evidence that the observed modulation is real.

The period of the optical/IR modulations seen in the GROND data for the Source 3 candidate counterpart is consistent with that seen in the XMM-Newton data for Source 3 (0.2245 \pm 0.0081 days; Section 3.1.3), and so the detection of virtually the same periodicity clearly indicates that the two objects are associated. Therefore, based on the X-ray and optical variability at the same period and the relatively low chance coincidence probability, we regard the association between Source 3 and its GROND candidate counterpart as robust. Furthermore, since we based the selection of candidate X-ray counterparts to 3FGL J2039.6-5618 on the search for variable sources, the optical/X-ray periodicity of Source 3 makes it a very promising candidate. Another possible candidate X-ray counterpart to 3FGL J2039.6-5618 would be Source 11, which is the second brightest $\mathrm{X}$-ray source detected in the 3FGL error circle, and the only other X-ray source with an obvious non-thermal spectrum (Table 1). However, Source 11 is not variable in X-rays and is not associated with a periodic optical/near-IR GROND counterpart. Therefore, although it cannot be firmly ruled out as a candidate X-ray counterpart to 3FGL J2039.6-5618, as of now, it is a less likely candidate by far than Source 3 . The same is true for all other fainter X-ray sources in Table 1, whose poorly characterized X-ray spectra and sparse optical, near-UV, and near-IR flux measurements hamper a detailed multiwavelength analysis of their properties and a non-ambiguous classification. 


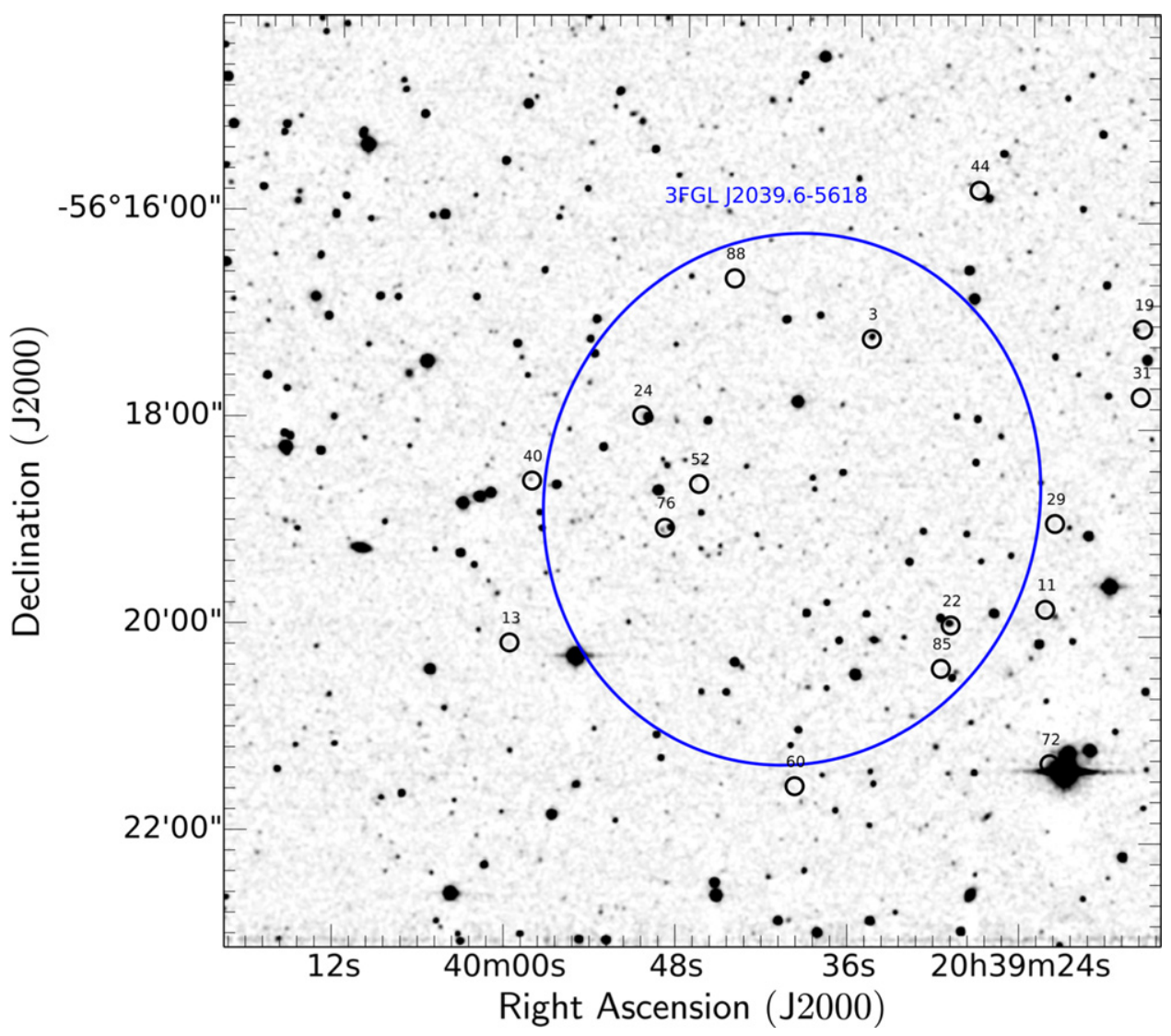

Figure 4. GROND J-band image of the 3FGL J2039.6-5618 field. The black circles indicate the positions of the XMM-Newton sources detected within, or close to, the 3FGL error ellipse, here represented by the blue ellipse. In all cases, the circle radius has been arbitrarily set to $5^{\prime \prime}$ for a better visualization.

\subsection{Characterization of the Source 3 Counterpart}

\subsubsection{Folded Light Curves Analysis}

The optical/near-IR light curves of the Source 3 counterpart folded at the corresponding best-fit periods are shown in Figure 5 (right). A double-peak light curve is clearly visible, with a main peak and a secondary peak separated in phase by $\sim 0.5$. For each band, we determined the phase of the two peaks by fitting a Gaussian function to their profiles to precisely compute their phase separations and errors. The peak phase separation remains constant in the optical $\left(0.436 \pm 0.003\right.$; $\left.\mathrm{g}^{\prime}\right)$, while it seems to slightly increase in the near-IR $(0.517 \pm 0.012 ; \mathrm{J})$. To better recognize the light curve evolution, we defined four regions: the main peak ( $\phi=0.2-0.5)$, the secondary peak $(\phi=0.7-0.9)$, the "bridge" $(\phi=0.5-0.7), \quad$ and the "off-peak" $(\phi=0.0-0.2$ and $\phi=0.9-1.0)$. The shape of the light curve is similar in all bands, but the profile of the modulation changes from the optical to the near-IR (Figure 6, left). In particular, the primary peak becomes broader and its amplitude decreases from $\sim 0.4 \mathrm{mag}$ in the $\mathrm{g}^{\prime}$ band to $\sim 0.2 \mathrm{mag}$ in the $\mathrm{H}$ band; the difference between the primary and secondary peaks decrease from $0.133 \mathrm{mag}$ in the $\mathrm{g}^{\prime}$ band to $\sim 0$ in the $\mathrm{H}$ band. Similarly, the amplitude of the "bridge" decreases, becoming comparable to that of the "off-peak" region. There is also possible evidence of a color variation as a function of phase (Figure 6, right), which might indicate that we are observing regions of the star surface at different temperatures. In particular, the colors seem to be bluer in coincidence with the two peaks, and bluer in coincidence with the primary peak than with secondary one.
The colors also seem bluer in the "bridge" than in the "offpeak" region. The color variation seems consistently less marked at longer wavelengths because the light curve variations are smoother. Unfortunately, the errors on the GROND photometry calibration (Section 2.3) make it difficult to quantify the observed color variations.

We tried to align in phase the X-ray and optical light curves of Source 3 to check the relative alignments between the X-ray and optical peaks. However, the difference between the epochs of the XMM-Newton and GROND observations $(\mathrm{MJD}=56885-56887$ and $\mathrm{MJD}=56575$, respectively) corresponds to a maximum time difference of 312 days. The accuracy on the best-fit optical period derived from the GROND observations is 0.00043 days (Section 3.2.2), which corresponds to a phase uncertainty of $\sim 0.0019$. Thus, building a phase-coherent solution for the optical light curve backward to the epoch of the XMM-Newton observation would bring an uncertainty of $\sim 0.59$ on the absolute phase determination, which is larger than the phase separation between the two peaks ( $\sim 0.3$ in the $\mathrm{X}$-rays and $\sim 0.45$ in the optical).

\subsubsection{Color-Magnitude Analysis}

We computed the time-average multi-band photometry of the Source 3 counterpart from the GROND data and obtained $\mathrm{g}^{\prime}=19.40 \pm 0.02, \quad \mathrm{r}^{\prime}=18.71 \pm 0.02, \quad \mathrm{i}^{\prime}=18.59 \pm 0.02$, $\mathrm{z}^{\prime}=18.52 \pm 0.02, \quad \mathrm{~J}=18.13 \pm 0.03, \quad \mathrm{H}=18.33 \pm 0.03$, and $\mathrm{K}=18.35 \pm 0.06$, with all magnitudes in the $\mathrm{AB}$ system. The photometry errors account for both statistical errors and the systematic uncertainty on the photometry calibration. We also 

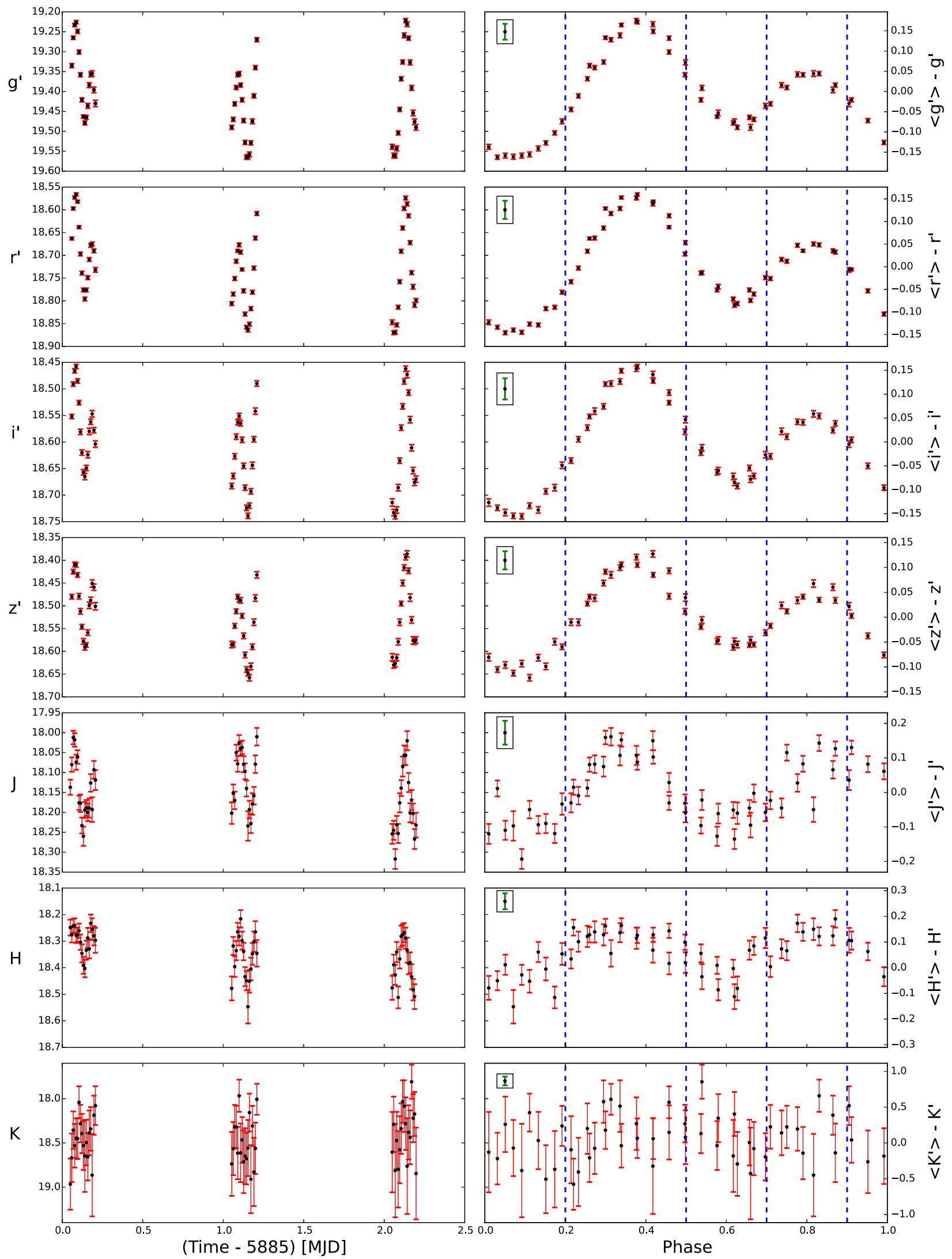

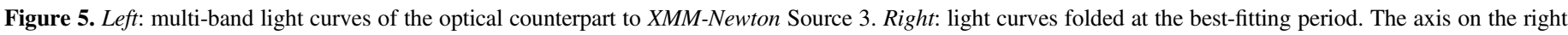

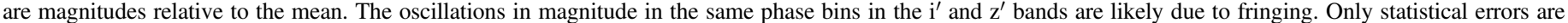

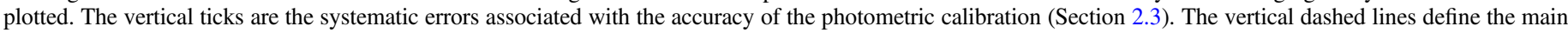
peak $(\phi=0.2-0.5)$, the secondary peak $(\phi=0.7-0.9)$, the "bridge" $(\phi=0.5-0.7)$, and the "off-peak" $(\phi=0.0-0.2$ and $\phi=0.9-1.0)$ regions. 

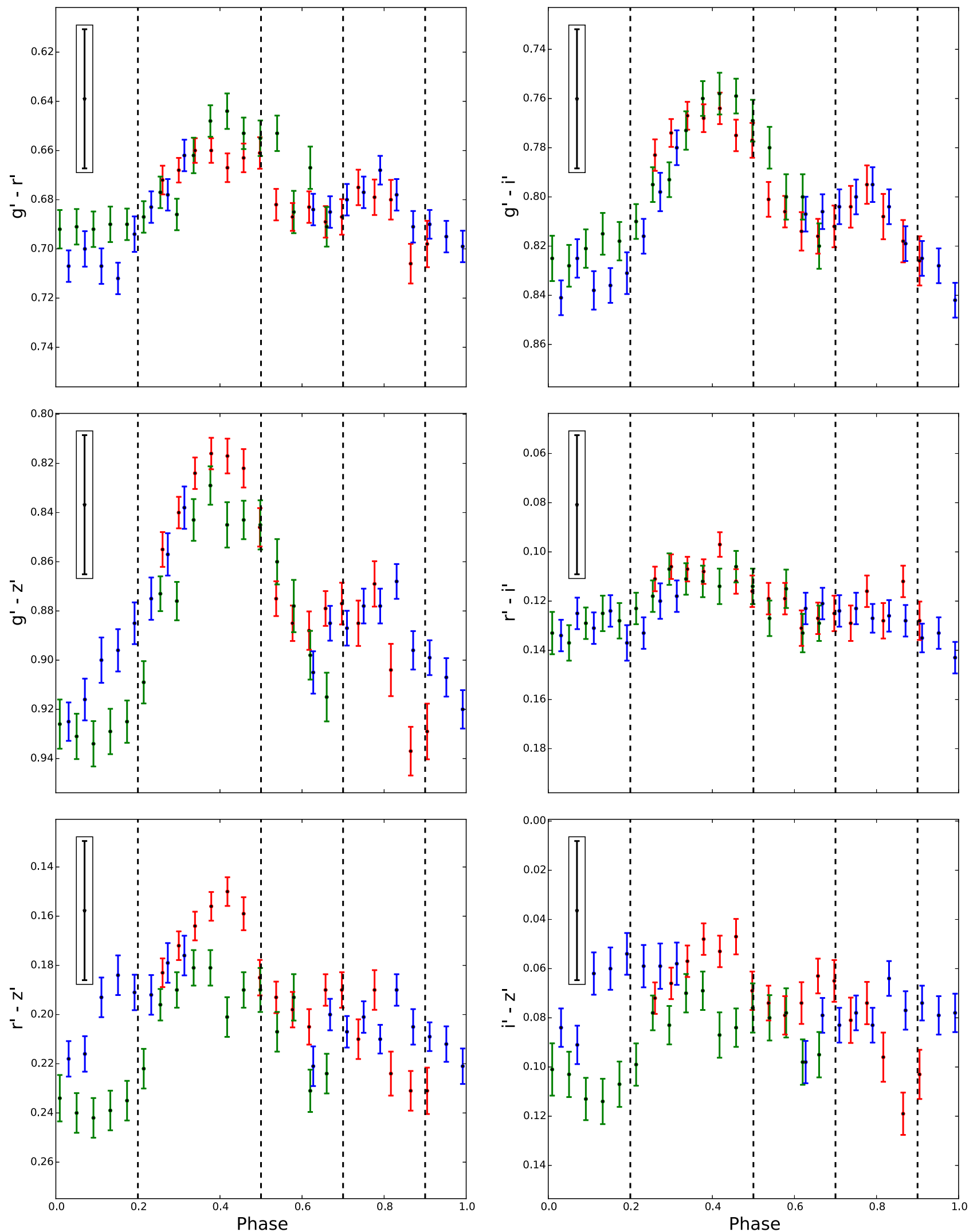

Figure 6. Colors of the Source 3 counterpart as a function of phase. Only statistical errors are plotted. The vertical ticks are the systematic errors in the color determination associated with the accuracy of the photometric calibration (Section 2.3). Different colors correspond to different nights (i.e., night 1, red; night 2, blue; night 3 , green). The vertical dashed lines correspond to the four regions defined in Figure 5. 
identified the Source 3 counterpart in the U and UVW1-filter exposures from the XMM-Newton/OM with $\mathrm{AB}$ magnitudes $\mathrm{U}=21.26 \pm 0.26$ and $m_{\mathrm{UVW} 1}=21.88 \pm 0.4$ (Table 2), whereas it is not detected in the UVM2 filter down to a $3 \sigma$ limiting magnitude of 21.99 and in the Swift/UVOT UVW2 image down to a limiting magnitude of 23.34 (AB). In the near-IR, we also identified the Source 3 counterpart in the VISTA images, with $\mathrm{AB}$ magnitudes $\mathrm{J}=18.24 \pm 0.03$, $\mathrm{H}=18.24 \pm 0.04$, and $\mathrm{K}_{\mathrm{s}}=18.64 \pm 0.09$, converted from the native Vega survey system. ${ }^{14}$ We found that the VISTA magnitudes (epoch 2010.6) are all compatible with the GROND ones (epoch 2014.7), after accounting for the difference between the $\mathrm{K}$ and $\mathrm{K}_{\mathrm{s}}$ filters, which excludes longterm variability on year timescales from the Source 3 counterpart.

We used the time-averaged $\mathrm{g}^{\prime}, \mathrm{r}^{\prime}, \mathrm{i}^{\prime}$, and $\mathrm{z}^{\prime}$-band magnitudes of the Source 3 counterpart as a reference for its classification by analyzing its location in the observed (i.e., not corrected for the reddening) color-magnitude (CM) and color-color (CC) diagrams of the field. The diagrams are shown in Figure 7, where the location of the field stars is shown by the black filled circles and that of the Source 3 counterpart as a red filled triangle. Red and green triangles correspond to the location computed from the single-image photometry. In order to reject outliers and include only high-confidence measurements, we plotted only field stars for which at least 20 measurements per filter were available and with $\sigma<0.08$. We compared the observed CM and CC diagrams with simulated stellar sequences computed from the Besançon models (Robin et al. 2004) for different stellar populations and distance values up to $15 \mathrm{kpc}$. The simulated sequences are shown in Figure 7 as the grayscale map. The dark gray regions in the CM diagrams correspond to a likely distance range $(200 \lesssim d \lesssim 900 \mathrm{pc})$ for Source 3, whereas in the CC diagram the dark gray region corresponds to simulated magnitudes that are within \pm 0.05 the $\mathrm{g}^{\prime}$-band magnitude of the Source 3 counterpart.

The distance to Source 3 is unknown a priori. The upper limit on the hydrogen column density derived from the fits to the X-ray spectrum of Source $3\left(N_{\mathrm{H}}<4 \times 10^{20} \mathrm{~cm}^{-2}\right.$; Table 1) indicates a distance lower than $\approx 0.9 \mathrm{kpc}$, assuming the relation between distance and $N_{\mathrm{H}}$ used by He et al. (2013). Without a parallax measurement, the distance to Source 3 cannot be precisely constrained from kinematic measurements of its optical counterpart. The NOMAD catalog (Zacharias et al. 2005) gives a proper motion of $\mu_{\alpha} \cos$ $(\delta)=14 \pm 4 \mathrm{mas} \mathrm{yr}^{-1}$ and $\mu_{\delta}=-16 \pm 9 \mathrm{mas} \mathrm{yr}^{-1}$ in right ascension and declination, respectively. This corresponds to a spatial velocity of $101_{-30}^{+32} \times(d / 1 \mathrm{kpc}) \mathrm{km} \mathrm{s}^{-1}$. If we equate it to the median of the transverse velocity distribution of MSPs $\left(\sim 108 \mathrm{~km} \mathrm{~s}^{-1}\right.$, with a standard deviation of $\left.\sim 86 \mathrm{~km} \mathrm{~s}^{-1}\right)$ computed from the Australia National Telescope Facility (ATNF) Pulsar Catalog ${ }^{15}$ (Manchester et al. 2005), we obtain a distance of $\sim 770-1500 \mathrm{pc}$ that is compatible with the estimate inferred from $N_{\mathrm{H}}$. Any determination of a lower limit on the distance is more uncertain. Again, if Source 3 were a binary MSP, the distance distribution of known binary MSPs from the ATNF Pulsar Catalog gives a probability of $\sim 0.006$ to find one within a radius of $0.2 \mathrm{kpc}$. Thus, the assumed distance range for Source $3(200 \lesssim d \lesssim 900 \mathrm{pc})$ is reasonable.

\footnotetext{
${ }^{14}$ http://casu.ast.cam.ac.uk/surveys-projects/vista/technical/filter-set

15 http://www.atnf.csiro.au/people/pulsar/psrcat
}

We also used the upper limit on the $N_{\mathrm{H}}$ to infer an interstellar extinction along the line of sight $E(B-V)<0.072$, after applying the relation of Predehl \& Schmitt (1995). Then, we computed the extinction in the different filters using the extinction coefficients of Fitzpatrick (1999). The reddening vectors are shown in Figure 7 for the limit case $E(B-V)=0.072$. Since the field stars are affected by an unknown interstellar extinction, and the simulations based on the Besançon models simply compute a reddening scaled proportionally to the assumed distance in a given direction, introducing a reddening correction in our simulations might bias a direct comparison between the observed and the simulated stellar sequences. Therefore, for simplicity, in all cases we simulated the stellar sequences assuming a null reddening. Then, we used the reddening vectors as a reference to trace the extinction-corrected locations of the observed points for the Source 3 counterpart (red points) along the simulated stellar sequences. As seen, the location of the Source 3 counterpart in the diagrams falls between the regions of the simulated MS and WD stellar sequences. Only for distances as low as $\sim 0.1 \mathrm{kpc}$ would the counterpart location in the diagrams be compatible with a WD. However, if Source 3 is an MSP, this possibility is unlikely (see above). Thus, we conclude that the star is most likely not a WD.

\subsubsection{Spectral Analysis}

We built the optical/near-UV/near-IR spectrum of the Source 3 counterpart using the available multi-band photometry (Section 3.3.2). In all cases, we used the measured $A B$ magnitudes as a reference to compute the spectral fluxes at the filter peak wavelengths. In addition, as a reference for the interstellar extinction correction, we used a maximum extinction value of $E(B-V)=0.072$ that is derived from the upper limit on the hydrogen column density $N_{\mathrm{H}}$ (Predehl \& Schmitt 1995) obtained with the fit with a PL spectral model (Section 3.1.2).

We fit the spectrum with both a single- and a double-BB spectral model. However, we found that the optical/near-UV/ near-IR data cannot be simultaneously fitted by a single BB and that a double $\mathrm{BB}$ is required to fit the entire spectrum $\left(\chi^{2}=20.6\right.$, 6 dof). The inferred temperatures are $T_{\mathrm{H}} \sim 3700 \mathrm{~K}$ for the hotter $\mathrm{BB}$, which fits the optical/UV fluxes, and $T_{\mathrm{C}} \sim 1600 \mathrm{~K}$ for the colder one, which fits the near-IR part of the spectrum. The double-BB model is also consistent with the upper limits obtained in the OM/UVW2 and UVOT/UVM2 filters. The overall spectral shape and spectral parameters do not change significantly when adding the correction for the maximum estimated interstellar extinction $\left(T_{\mathrm{H}} \sim 3800 \mathrm{~K}, T_{\mathrm{C}} \sim 1600 \mathrm{~K}\right)$. While the temperature of the hot $\mathrm{BB}$ is compatible with the surface temperature of a mid-MS companion star, the temperature of the colder one is too low to be entirely ascribed to the emission from the star surface. Although this can contribute to part of the observed near-IR emission, as indicated by the periodic modulation of the $\mathrm{J}-, \mathrm{H}-$, and $\mathrm{K}$-band light curves, an additional source external to the star is required to account for the low temperature of the BB that fits the near-IR part of the spectrum. This source might be associated with emission from cold intrabinary gas or dust, may be the residual of an accretion disk left over by a past phase of matter accretion on the neutron star.

The analysis of the counterpart colors as a function of phase (Figure 6, right) suggests that its spectrum slightly changes along the $\sim 0.2245$ days period. To quantify this possible 

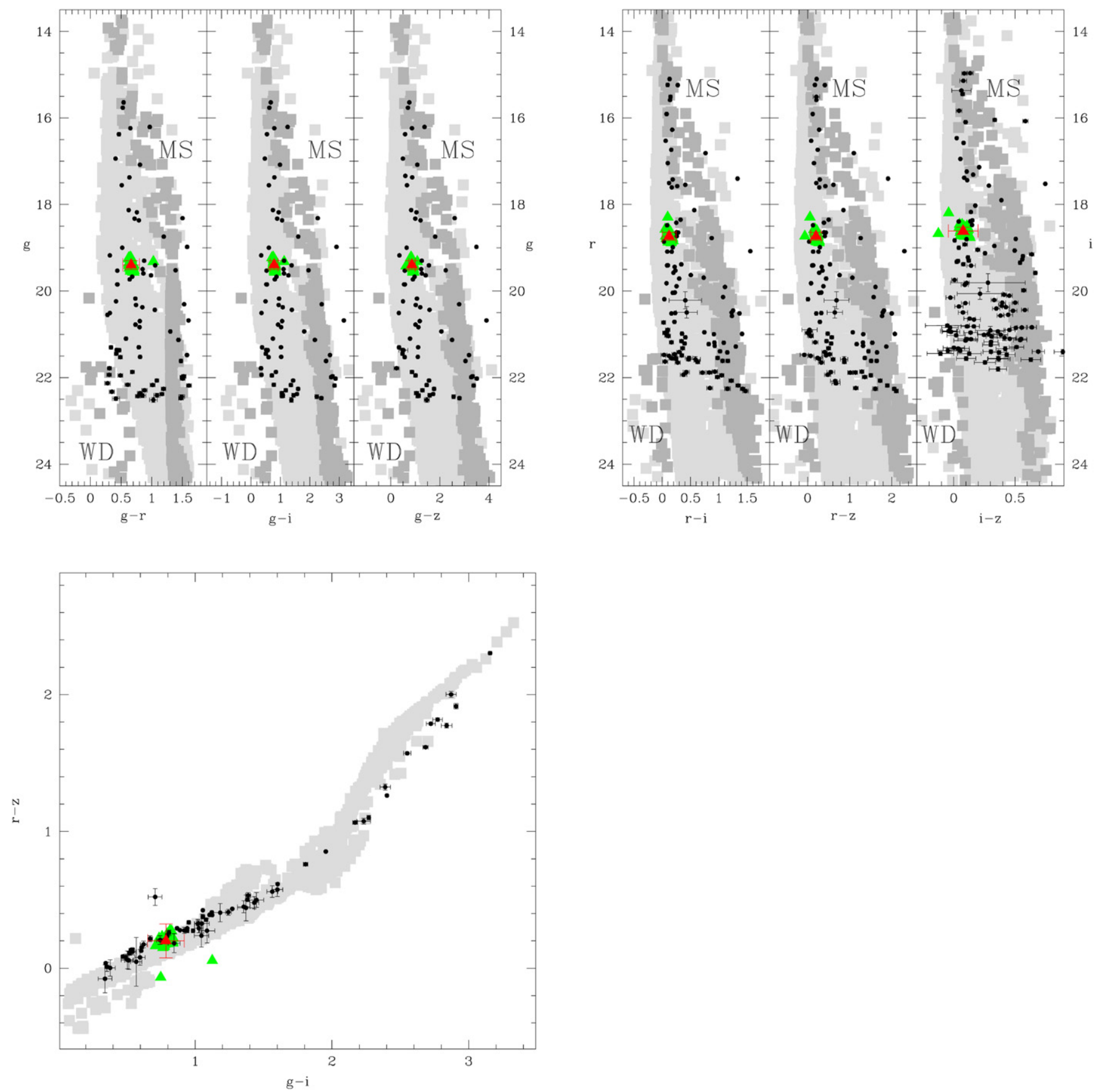

Figure 7. Top: observed CMDs for the 3FGL J2039.6-5618 field obtained from the GROND time-averaged photometry. Bottom: observed CC diagram. In all panels, the location of field stars is indicated by the black filled circles, whereas that of the optical counterpart of the XMM-Newton Source 3 is indicated by the red filled triangle. The filled green triangles indicate the counterpart location computed from the photometry computed on the single image. Stellar sequences simulated from the Besançon models for different values of distance are shown in light and dark gray. In the CM diagrams the dark gray regions correspond to distance values $200<d<900 \mathrm{pc}$, whereas in the CC diagram they correspond to magnitudes within \pm 0.05 the $\mathrm{g}^{\prime}$-band magnitude of the Source 3 counterpart. The MS and WD branches are labelled.

evolution, we fitted the multi-band spectrum in the four phase intervals defined in Section 3.3.1. Like in our phase-resolved color analysis, we cannot use the single-epoch flux measurements in the U and UVW1 bands obtained with the XMMNewton/OM. As mentioned previously, we fitted the four phase-resolved spectra using a double-BB model, considering both null and maximum interstellar extinction. However, we did not find evidence of a significant spectrum evolution across the different phase intervals. This is partially ascribed to the fact the spectra are less constrained at shorter wavelengths without the flux measurements in the U and UVW1 bands.

\section{DISCUSSION}

The optical and X-ray emission of Source 3, modulated at a common periodicity of $\sim 0.2245$ days, is likely associated with the orbital motion of a tight binary system. The IR/optical/UV spectrum points at a late spectral type (K or M) for at least one of the stars in the system (Section 3.3.3). The other object may 


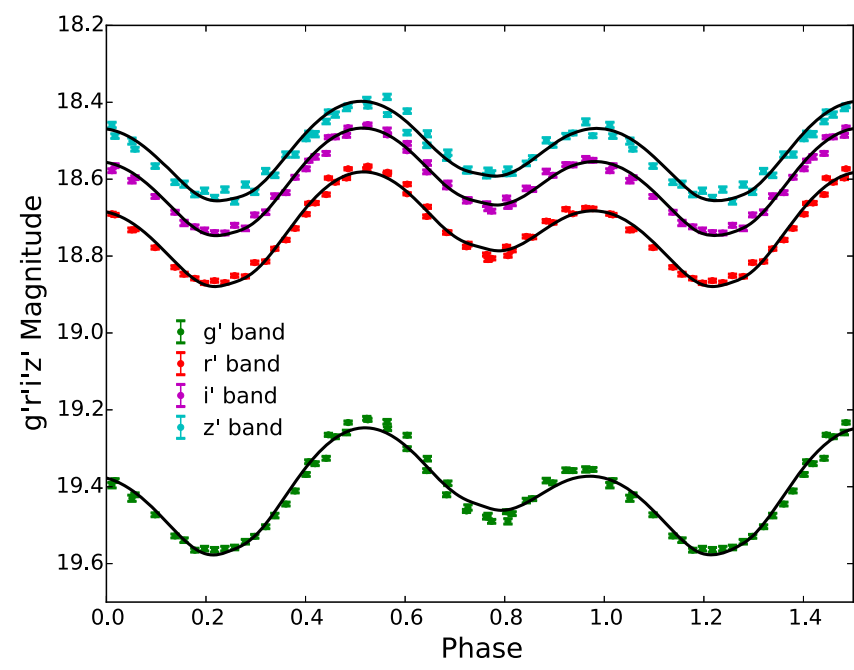

Figure 8. Multi-band light curve of the optical counterpart of the XMM-Newton Source 3. The $\mathrm{g}^{\prime}, \mathrm{r}^{\prime}, \mathrm{i}^{\prime}$ and $\mathrm{z}^{\prime}$ bands are marked by green, red, magenta, and cyan circles, respectively. Only statistical errors are plotted. The black lines display the best-fit light curve calculated using the model described in Section 4.1.

either be another non-degenerate star or a compact object, likely an MSP. In the first scenario, the X-ray activity, the orbital period and the shape of the optical light curves indicate that the system could either be of the $\mathrm{W}$ UMa or $\beta$ Lyr type (Geske et al. 2006). However, the system would be extremely peculiar even for these classes of binaries. The orbital period would be one of the shortest ever observed, the spectral type one of the latest, and the asymmetry and separation of the peaks in the optical light curves hard to explain. The second scenario sounds more plausible. Moreover, when compared with the $\gamma$-ray flux of 3FGL J2039.6-5618, $F_{\gamma}=(1.71 \pm 0.14) \times 10^{-11} \mathrm{erg} \mathrm{cm}^{-2} \mathrm{~s}^{-1}$, the $0.3-10 \mathrm{keV}$ unabsorbed X-ray flux of Source 3 $\left(F_{\mathrm{X}}=10.19_{-0.82}^{+0.87} \times 10^{-14} \mathrm{erg} \mathrm{cm}^{-2} \mathrm{~s}^{-1}\right)$ would give a $\gamma$-to-Xray flux ratio of $F_{\gamma} / F_{\mathrm{X}} \approx 170$, which is consistent with that of MSPs (Abdo et al. 2013; Marelli et al. 2015). 3FGL J2039.6 -5618 is also at a relatively high Galactic latitude, with $l=341^{\circ} .23$ and $b=-37^{\circ} .15$, like most MSPs. We note that the NOMAD proper motion of Source 3 in Galactic coordinates, $\mu_{l}=-17 \pm 4 \mathrm{mas} \mathrm{yr}^{-1}$ and

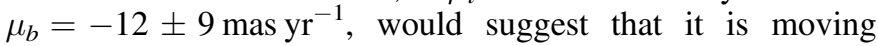
toward the Galactic center from its present location. This, however, would not argue against an MSP identification because several MSPs in the ATNF catalog have a negative proper motion in Galactic latitude. Being older than a Gyr, MSPs are indeed expected to orbit in the Galactic potential and periodically move away and toward the plane. Interestingly, if ascribed to an orbital motion, the period of the observed optical flux modulation ( $\sim 0.2245$ days) would be comparable to the orbital periods of BW and RB MSPs, which are usually less than a day (see, e.g., Roberts 2013). Because most binary MSPs detected as $\gamma$-ray pulsars by the Fermi-LAT are either BWs or RBs, the possible identification of Source 3 as a BW/ RB system would, then, concur to make it the most likely X-ray counterpart to 3FGL J2039.6-5618.

The X-ray emission model for BW and RB is generally described by a the combination of a thermal component, which originates from the neutron star surface, and a non-thermal component, which primarily originates from an intrabinary shock and from the neutron star magnetosphere (Gentile et al. 2014). The X-ray spectrum of Source 3 is predominantly non-thermal, with a photon index $\Gamma_{\mathrm{X}}=1.36$ and its $\mathrm{X}$-ray luminosity in the $0.3-10 \mathrm{keV}$ energy band is $L_{\mathrm{X}} \sim 10^{31} \mathrm{erg} \mathrm{s}^{-1} d_{\mathrm{kpc}}^{2}$, where $d_{\mathrm{kpc}}$ is the distance in units of kpc. Both the X-ray luminosity and photon index of Source 3 are in general agreement with those of RB/BW MSPs (Gentile et al. 2014; Roberts et al. 2014), although its relatively hard X-ray spectrum would point more at an RB than a BW. As we noted in Section 3.1.2, a thermal component might be present in the X-ray spectrum of Source 3. However, further observations are necessary to clearly discriminate between a purely non-thermal and a composite spectral model.

The X-ray light curve of Source 3 can be explained assuming a binary MSP scenario; the emission from the intrabinary shock is expected to be modulated at the orbital period. Recent studies suggest that the X-ray modulation may be due to synchrotron beaming, Doppler boosting of the flow within the shock, or obscuration by the companion (Bogdanov et al. 2011; Gentile et al. 2014; Roberts et al. 2014). The shape of the X-ray light curve strongly depends on the geometrical and physical parameters of the binary system, but, on average, it is characterized by an overall increase of a factor of $\sim 2-3$ around inferior conjunction. The light curves of many BWs and RBs show a double-peaked structure due to the obscuration of part of the shock by the companion around the superior conjunction. The inclination angle of the system, as well as the ratio of the companion radius to the intrabinary separation, characterize the phase separation and levels of the two peaks. The X-ray light curve of Source 3 clearly points at a BW/RB scenario. Its amplitude changes by a factor of $\sim 3$ during the orbit; the minima occur at orbital phases $0.0-0.1$ and $0.9-0.0$, which correspond to the superior conjunction, whereas the maxima occur at phase $0.4-0.7$, which corresponds to the inferior conjunction. Unfortunately, we cannot align in phase $\mathrm{X}$-ray and optical light curves (Section 3.3.1) to confirm this scenario.

The optical emission in BWs and RBs is dominated by the non-degenerate companion and is characterized by significant luminosity and color variations. The optical light curve profile can bring the signature of two different effects: the tidal distortion of the companion star surface due to the gravitational pull of the MSP, and the heating of the companion star surface due to the irradiation of relativistic photons and/or high-energy particles from the MSP (Breton et al. 2013). Most BWs exhibit a single-peak optical light curve (e.g., Stappers et al. 2001) due to the irradiation of the nearly fully peeled companion star from the MSP. Half of the RBs also feature a single peak, whereas the rest feature a two-peaked light curve, with the peaks occurring at specific orbital phases due to the viewing geometry of the tidally distorted, non-degenerate companion star. In the latter case, the companion star nearly fills its Roche lobes and tidal distortion effects dominate over the heating effects. Examples of RBs with single-peak light curves include PSR J1023+0038 (Thorstensen \& Armstrong 2005), J2215+5135 (Schroeder \& Halpern 2014), J2339-0533 (Romani \& Shaw 2011), and J1227-4853 (Bassa et al. 2014), whereas examples of RBs with double-peak light curves include PSR J1628-3205 (Li et al. 2014), J1723-2837 (van Staden et al. 2015), J1816+4510 (Kaplan et al. 2012), and J2129 -0428 (Hui et al. 2015). The double-peak light curves of the RBs look very similar to that of Source 3, displaying 
asymmetries in the relative phase of the two peaks. In particular, the optical light curve of PSR J1628-3205 (Li et al. 2014) shows the same remarkable asymmetry between the two peaks, with a main and secondary peak, as observed in the Source 3 counterpart. This similarity would then support the hypothesis that Source 3 is a RB.

The $\gamma$-ray error ellipse of 3FGL J2039.6-5618 has been observed four times at the CSIRO Parkes telescope searching for pulsations up to a $D M=200 \mathrm{pc} \mathrm{cm}^{-3}$ (Camilo et al. 2015). No pulsations were found, but this is not unexpected because RBs are very elusive targets in radio. In fact, the intrabinary material ablated from the star causes strong and variable scattering and absorption of radio waves. The radio detection of the pulsations often requires several dedicated long observations (Ray et al. 2013; P. Ray et al. 2015, in preparation).

\subsection{Modeling of the Optical Light Curve}

Standard models for the RB optical light curves based on tidal distortion and pulsar irradiation (e.g., Thorstensen \& Armstrong 2005) cannot fit well with either the asymmetric peaks or the peak separation. Therefore, we built a simple three-dimensional model, including an additional component related to the asymmetric irradiation of the companion star to fit the light curve of the Source 3 counterpart. In this process, we considered only the optical light curves because they have the highest signal-to-noise.

In order to probe the RB scenario and estimate its physical parameters, we built a simple three-dimensional model of a RB binary system with very few free parameters, and fit it to the observed optical light curves of Source 3. In this model, the shape of the companion star is approximated by a sphere and a tangent cone pointing to the neutron star. The cone is meant to account for the tidal deformation of the star as it approaches filling its Roche lobe. By locking the star rotation to the orbital motion, we assumed two different brightnesses for night and day, a characteristic commonly found in RB systems. The asymmetry in the two peaks of the optical light curve implies some asymmetry in the physical system that produces it. Therefore, we allowed a tilt angle between the cone axis and the day/night separator line, as measured on the orbital plane. As for other RBs, we assumed a perfectly circular orbit, reducing the number of free parameters in Kepler's orbital parameter space. To summarize, our model accounts for four geometrical parameters: the star deformation (the distance from the cone tip to the star center in star radius units), the night/day asymmetry (the angle between the cone axis and the line of sight to the star), the orbital inclination (the angle between the line of sight and the orbital plane), and the epoch of quadrature (when the projected axis of companion star orbit lies perpendicular to the day/night seperator line). Furthermore, for each band we have two free parameters: the light curve normalization and the brightness ratio between night and day.

We fit our model to the data (Figure 8), including both statistical and systematic errors, and found good qualitative agreement for a narrow range of parameters. The overall goodness of fit turns out to be 197.3 (196 dof). The configuration obtained from the best-fit to the model implies a deformation of $1.519 \pm 0.009$ for the companion star, indicating that it is subject to strong tidal effects, and a large value of the asymmetry, $52^{\circ} .1 \pm 1.2$. The orbital inclination of the binary system obtained from the best-fit is $48^{\circ} .9 \pm 0.6$, whereas the epoch of quadrature is at MJD
$56884.9667 \pm 0.0003$. Finally, the best-fit brightness ratios between the night and day sides in the four bands are $0.789 \pm 0.007\left[\mathrm{~g}^{\prime}\right], 0.828 \pm 0.007\left[\mathrm{r}^{\prime}\right], 0.851 \pm 0.007\left[\mathrm{i}^{\prime}\right]$, and $0.878 \pm 0.008\left[\mathrm{z}^{\prime}\right]$

The model assumes, as a first approximation, that the night and day sides of the star are in local thermodynamical equilibrium at different temperatures: $T_{\text {night }}$ and $T_{\text {day }}$, respectively. A brightness ratio implies a relation between these two temperatures, as a function of the temperature itself. This relation weakly depends on the interstellar extinction, which can only be constrained by our upper limits on the $N_{\mathrm{H}}$. We computed these relations for each band, adding in quadrature the uncertainty associated with the interstellar extinction correction. The values of temperature ratios are compatible in the four bands, at $1 \sigma$, only for a value of the day-side temperature $T_{\text {day }}<9900 \mathrm{~K}$. This constraint does not rely on the normalizations, hence on the accuracy of the photometric calibration. It is a side product of our model that is consistent with the spectral results obtained in Section 3.3.3. On the other hand, the model implies a simple relation between the temperature ratio and their absolute value:

$$
\begin{aligned}
\frac{T_{\text {day }}-T_{\text {night }}}{T_{\text {day }}}= & 8.666 \times 10^{-3}\left(\frac{T_{\text {day }}}{1000 \mathrm{~K}}\right) \\
& -1.890 \times 10^{-4}\left(\frac{T_{\text {day }}}{1000 \mathrm{~K}}\right)^{2} .
\end{aligned}
$$

The large value of the asymmetry, $\sim 50^{\circ}$, is implied by the different levels of minima and maxima in the light curves. Similar features are observed in other RBs, such as PSR J1628 -3205 (Li et al. 2014), where asymmetry was also proposed as an explanation. In those cases, a measure of this effect was not possible using the standard modeling tools, which motivated our alternative modeling. In our model, an asymmetry causes a phase shift between the sinusoid that represents the day/night variation and the peaks due to the bump. If the brighter temperature is induced by irradiation from the neutron star, this must be channeled through wind rather than photons. In fact, high-energy radiation travels straight lines, implying a symmetric heating, while particles may follow other trajectories. Alternatively, the apparent difference in temperature could be due to very large spots on the surface of the low-mass star. This would not be totally unexpected, given the purely convective nature of this kind of stars, and the perturbation induced by the pulsar. If this were the case, a future observation may reveal a different asymmetry, indicating a migration of the spots on the surface.

\section{CONCLUSIONS}

We carried out multi-wavelength observations of the unidentified Fermi-LAT source 3FGL J2039.6-5618 with XMM-Newton and GROND. We detected a likely X-ray counterpart (Source 3) within the $\gamma$-ray error box of 3FGL J2039.6-5618, which is characterized by a PL X-ray spectrum $\left(\Gamma_{\mathrm{X}}=1.36 \pm 0.09\right)$ that is indicative of strong magnetospheric emission. The upper limit on the hydrogen column density inferred from the X-ray spectral fit, $N_{\mathrm{H}}<4 \times 10^{20} \mathrm{~cm}^{-2}$, implies a distance that is probably lower than $1 \mathrm{kpc}$. The X-ray light curve of Source 3 features a modulation with a period of $0.2245 \pm 0.0081$ days and two peaks, separated in phase by $\sim 0.3$. Using the GROND data, we found an optical counterpart to Source 3 that features an asymmetric, double-peaked 
light curve and a flux modulation with a period of $0.22748 \pm 0.00043$ days, which is coincident with that measured in the X-rays. If we interpret this periodicity as the orbital period of a compact binary system, Source 3 would probably be a binary MSP and, as such, a very likely counterpart to 3FGL J2039.6-5618. In particular, both of the optical colors of the putative companion star and the optical light curve profile, with two asymmetric peaks separated in phase by $\sim 0.5$, suggest that Source 3/3FGL J2039.6-5618 is a RB. This hypothesis is supported by the lack of apparent radio emission, which would be explained by the eclipse of the radio signal as it propagates through the evaporated atmosphere of the irradiated MSP companion, as observed in many RBs.

Optical spectroscopy observations of Source 3 will be essential to obtain a more accurate classification of its companion star and measure its radial velocity curve, which are crucial to confirm the binary MSP scenario and determine the orbital parameters of the binary system. A precise determination of the orbital parameters is necessary to fold $\gamma$ ray photons and search for the MSP pulsations. A timing solution, which can be extended back in time to the launch of Fermi in 2008, will provide even tighter constraints on the orbital parameters and their evolution. This has a potentially huge scientific payoff in terms of fundamental physics (Romani et al. 2012; Pletsch \& Clark 2015).

The research leading to these results has received funding from the European Commission Seventh Framework Programme (FP7/2007-2013) under grant agreement n. 267251. This work was supported by the ASI-INAF contract I/004/11/ 0, art.22 L.240/2010 for the project "Studio di sorgenti di alta energia con Swift." C.D. acknowledges support through EXTraS, funded from the European Union's Seventh Framework Programme for research, technological development, and demonstration under grant agreement no 607452. Part of the funding for GROND (both hardware as well as personnel) was generously granted from the Leibniz-Prize to Prof. G. Hasinger (DFG grant HA 1850/28-1).

Note added in proof. After our manuscript was submitted, we became aware of an independent analysis of our XMMNewton and GROND data carried out by Romani (2015) soon after they became public, which is in line with our results.

Facilities: XMM-Newton, Max Planck: 2.2m (GROND), Swift, Suzaku, VISTA.

\section{REFERENCES}

Abdo, A. A., Ackermann, M., Ajello, M., et al. 2009, Sci, 325, 840 Abdo, A. A., Ackermann, M., Ajello, M., et al. 2010, ApJS, 188, 405 Abdo, A. A., Ajello, M., Allafort, A., et al. 2013, ApJS, 208, 17 Acero, F., Ackermann, M., Ajello, M., et al. 2015, ApJS, 218, 23 Ackermann, M., Ajello, M., Allafort, A., et al. 2012, ApJ, 753, 83 Atwood, W. B., Ziegler, M., Johnson, R. P., \& Baughman, B. M. 2006, ApJ, 652,49

Atwood, W. B., Abdo, A. A., Ackermann, M., et al. 2009, ApJ, 697, 1071

Bassa, C. G., Patruno, A., Hessels, J. W. T., et al. 2014, MNRAS, 441, 1825 Bevington, P. R. 1969, Data Reduction and Error Analysis for the Physical Science (New York: McGraw-Hill)

Bogdanov, S., Archibald, A. M., Hessels, J. W. T., et al. 2011, ApJ, 742, 97 Breeveld, A. A., Curran, P. A., Hoversten, E. A., et al. 2010, MNRAS, 406, 1687 Breton, R. P., van Kerkwijk, M. H., Roberts, M. S. E., et al. 2013, ApJ, 769, 108 Camilo, F., Kerr, M., Ray, P. S., et al. 2015, ApJ, 810, 85

Dalton, G. B., Lewis, I. J., Bonfield, D. G., et al. 2006, Proc. SPIE, 6269, 4
De Luca, A., Caraveo, P. A., Mereghetti, S., Negroni, M., \& Bignami, G. F. 2005, ApJ, 623, 1051

Dickey, J. M., \& Lockman, F. J. 1990, ARA\&A, 28, 215

Emerson, J., McPherson, A., \& Sutherland, W. 2006, Msngr, 126, 41

Emerson, J. P., Irwin, M. J., Lewis, J., et al. 2004, Proc. SPIE, 5493, 401 Fitzpatrick, E. L. 1999, PASP, 111, 63

Gentile, P. A., Roberts, M. S. E., McLaughlin, M. A., et al. 2014, ApJ, 783, 69 Gilliland, R. L., \& Baliunas, S. L. 1987, ApJ, 314, 766

Geske, M. T., Gettel, S. J., \& McKay, T. A. 2006, ApJ, 131, 633

Greiner, J., Bornemann, W., Clemens, C., et al. 2008, PASP, 120, 405

He, C., Ng, C.-Y., \& Kaspi, V. M. 2013, ApJ, 768, 64

Hui, C. Y., Hu, C. P., Park, S. M., et al. 2015, ApJL, 801, 27

Kaplan, D. L., Stovall, K., Ransom, S. M., et al. 2012, ApJ, 753, 174

Kataoka, J., Yatsu, Y., Kawai, N., et al. 2012, ApJ, 757, 176

Kong, A. K. H., Huang, R. H. H., Cheng, K. S., et al. 2012, ApJL, 747, L3

Kong, A. K. H., Jin, R., Yen, T.-C., et al. 2014, ApJL, 794, L22

Krühler, T., et al. 2008, ApJ, 685, 376

Leahy, D. A. 1987, A\&A, 180, 275

Lee, K. J., Guillemot, L., Yue, Y. L., Kramer, M., \& Champion, D. J. 2012, MNRAS, 424, 2832

Lenz, P., \& Breger, M. 2005, CoAst, 146, 53

Li, M., Halpern, J. P., \& Thorstensen, J. R. 2014, ApJ, 795, 115

Lomb, N. R. 1976, ApSS, 39, 447

Manchester, R. N., Hobbs, G. B., Teoh, A., \& Hobbs, M. 2005, AJ, 129, 1993

Marelli, M., Harding, H., Pizzocaro, D., et al. 2014, ApJ, 795, 168

Marelli, M., Mignani, R. P., De Luca, A., et al. 2015, ApJ, 802, 78

Mason, K. O., Breeveld, A., Much, R., et al. 2001, A\&A, 365, 36

Mauch, T., Murphy, T., Buttery, H. J., et al. 2003, MNRAS, 342, 1117

Mirabal, N., Frías-Martinez, V., Hassan, T., \& Frías-Martinez, E. 2012, MNRAS, 424, 64

Mitsuda, K., Bautz, M., Inoue, H., et al. 2007, PASJ, 59, 1

Monet, D. G., Levine, S. E., Canzian, B., et al. 2003, AJ, 125, 984

Nolan, P. L., Abdo, A. A., Ackermann, M., et al. 2012, ApJS, 199, 31

Page, M. J., Brindle, C., Talavera, A., et al. 2012, MNRAS, 426, 903

Petrov, L., Mahony, E. K., Edwards, P. G., et al. 2013, MNRAS, 432, 1294

Pletsch, H. J., Guillemot, L., Fehrmann, H., et al. 2012, Sci, 338, 1314

Pletsch, H. J., Guillemot, L., Allen, B., et al. 2013, ApJL, 779, L11

Pletsch, H. J., \& Clark, C. J. 2014, ApJ, 795, 75

Pletsch, H. J., \& Clark, C. J. 2015, ApJ, 807, 718

Poole, T. S., Breeveld, A. A., Page, M. J., et al. 2008, MNRAS, 383, 627

Predehl, P., \& Schmitt, J. H. M. M. 1995, A\&A, 293, 889

Ray, P. S., Ransom, S. M., Cheung, C. C., et al. 2013, ApJL, 763, L13

Roberts, M. S. E. 2013, in IAU Symp. 291 (Cambridge: Cambridge Univ. Press), 127

Roberts, M. S. E., McLaughlin, M. A., Gentile, P., et al. 2014, AN, 335,313

Robin, A. C., Reylé, C., Derriére, S., \& Picaud, S. 2004, A\&A, 416, 157

Romani, R. W. 2015, ApJL, 812, L24

Romani, R. W., Filippenko, A. V., \& Cenko, S. B. 2014, ApJL, 793, L20

Romani, R. W., Filippenko, A. V., \& Silverman, J. M. 2012b, ApJL, 760, L36

Romani, R. W., \& Shaw, M. S. 2011, ApJL, 743, L26

Roming, P. W. A., Kennedy, T. E., Mason, K. O., et al. 2005, Space Sci. Rev., 120,95

Salvetti, D. \& Fermi-LAT Collaboration 2013, American Astronomical Society HEAD Meeting 13, \#117.07

Scargle, J. D. 1982, ApJ, 302, 757

Schroeder, J., \& Halpern, J. 2014, ApJ, 793, 78

Skrutskie, M. F., Cutri, R. M., Stiening, R., et al. 2006, AJ, 131, 1163

Stappers, B. W., van Kerkwijk, M. H., Bell, J. F., \& Kulkarni, S. R. 2001, ApJL, 548, L183

Stellingwerf, R. F. 1978, ApJ, 224, 953

Strader, J., Chomiuk, L., Sonbas, E., et al. 2014, ApJL, 788, L27

Struder, L., Briel, U., Dennerl, K., et al. 2001, A\&A, 365, L18

Takeuchi, Y., Kataoka, J., Maeda, K., et al. 2013, ApJS, 208, 25

Thorstensen, J. R., \& Armstrong, E. 2005, AJ, 130, 759

Turner, M. J. L., Abbey, A., Arnaud, M., et al. 2001, A\&A, 365, 27

van Staden, A. 2015, MNSSA, 74, 22

Yoldas, A. K., et al. 2008, in AIP Conf. Proc. 1000, GAMMA-RAY BURSTS 2007: Proc. Santa Fe Conf. (Melville, NY: AIP), 227

York, D. G., Adelman, J., Anderson, J. E., Jr., et al. 2000, AJ, 120, 1579

Zacharias, N., Monet, D. G., Levine, S. E., et al. 2005, BAAS, 36, 1418

Zechmeister, M., \& Kuerted, M. 2009, A\&A, 496, 577 